\title{
A TESTBED FOR QOS-BASED DATA ANALYTIC SERVICE SELECTION IN THE CLOUD
}

by

Md Shahinur Rahman

B.Sc. in Computer Science, Khulna University of Engineering and Technology, Khulna, Bangladesh, 2003

\author{
A thesis \\ presented to Ryerson University \\ in partial fulfillment of the \\ requirements for the degree of \\ Master of Science \\ in the program of \\ Computer Science
}

Toronto, Ontario, Canada, 2015

CMd Shahinur Rahman 2015 


\section{AUTHOR'S DECLARATION}

I hereby declare that I am the sole author of this thesis. This is a true copy of the thesis, including any required final revisions, as accepted by my examiners.

I authorize Ryerson University to lend this thesis to other institutions or individuals for the purpose of scholarly research.

I further authorize Ryerson University to reproduce this thesis by photocopying or by other means, in total or in part, at the request of other institutions or individuals for the purpose of scholarly research.

I understand that my thesis may be made electronically available to the public. 


\title{
A TESTBED FOR QOS-BASED DATA ANALYTIC SERVICE SELECTION IN THE CLOUD
}

\author{
Md Shahinur Rahman \\ Master of Science, Computer Science, 2015 \\ Ryerson University
}

\begin{abstract}
With the emergence of Big Data and Cloud Computing, more and more data analytic software services have become available through a Cloud platform. Compared to the traditional service selection problem, selecting this type of services has additional challenges, which requires new selection models being proposed. It is the purpose of this work to "create a testbed" to benefit the research community in this area so that different selection models with consideration of different performance-influencing factors such as algorithms implemented, datasets to be processed, hosting infrastructure, can be tested and compared. We created a cloud-based platform for publishing and invoking analytic services as well as monitoring service performance during invocation. We implemented various data mining algorithms from different packages as example analytic services and hosted them on different infrastructure services. We also ran these services on some real datasets to collect a sample dataset of their Quality of Service (QoS) values.
\end{abstract}




\section{ACKNOWLEDGEMENTS}

I would like to express my sincere appreciation to my supervisor Dr. Cherie Ding for her valuable support and guidance in helping me to go through all the complexity in my work. Her valuable suggestions and guidance have greatly enhanced my knowledge and skills in research and have significantly contributed to the completion of this thesis.

I am very grateful to Dr. Alex Ferworn, Dr. Ali Miri and Dr. Andriy Miranskyy for taking time to review my thesis and provide their feedbacks.

In addition, I would like to thank all of my friends specially S.M. Rana and Md. Mizanur Rahman who have reviewed my thesis and have given me valuable comments which enabled me to improve my thesis.

Also, I would like to acknowledge the support of the Computer Science Department of Ryerson University and my fellow students.

Finally, I would like to express my deep appreciations to my family and relatives who have motivated and supported me during these years of study. 


\section{TABLE OF CONTENTS}

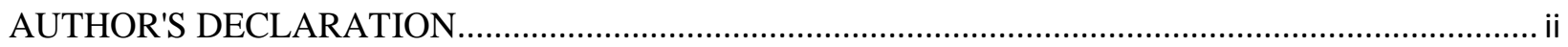

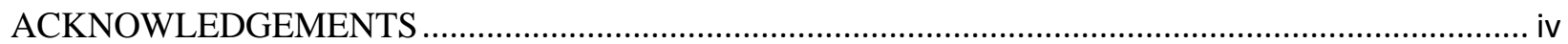

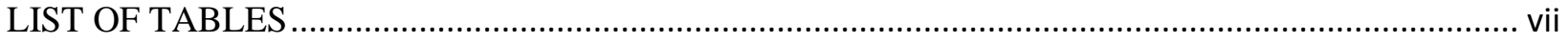

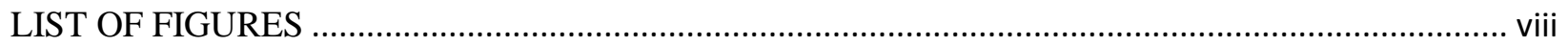

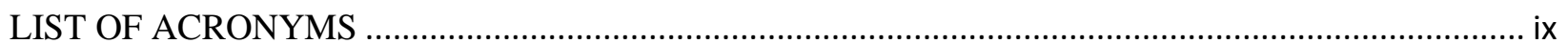

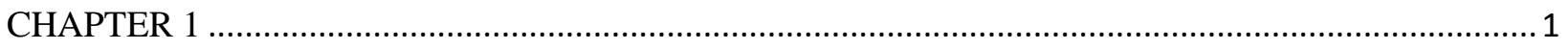

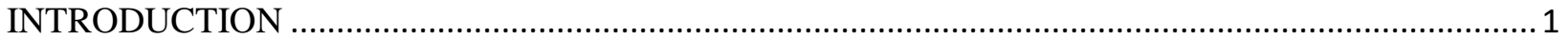

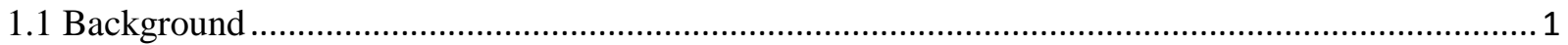

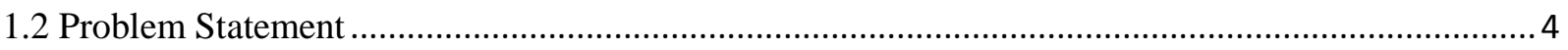

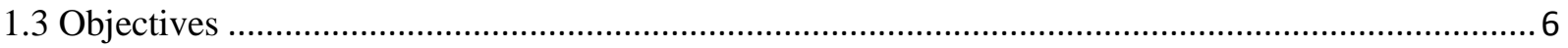

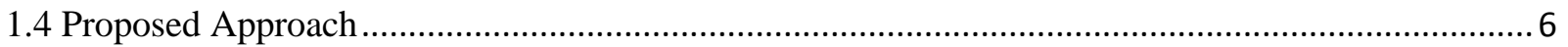

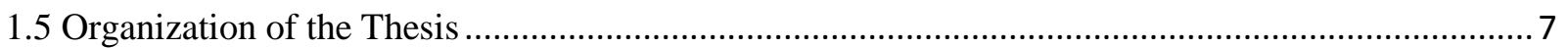

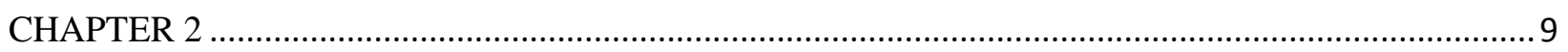

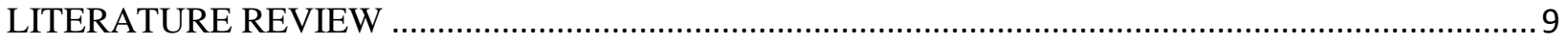

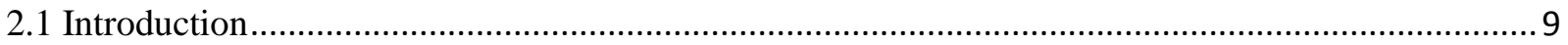

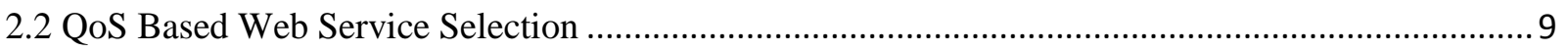

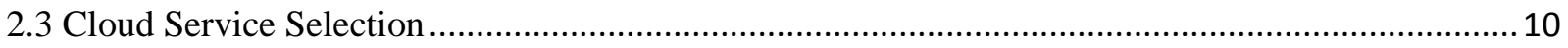

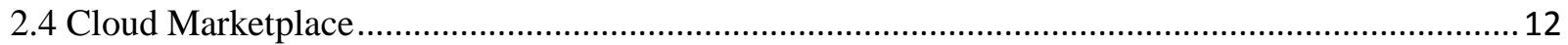

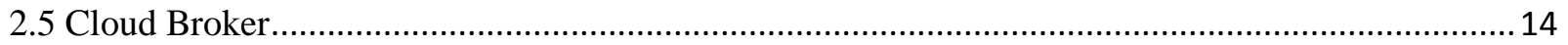

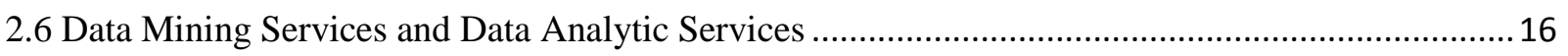

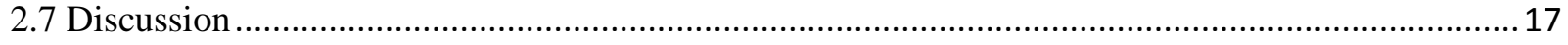

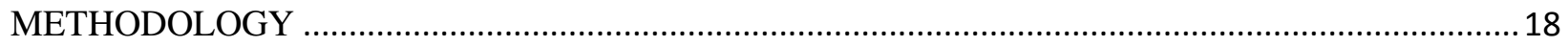

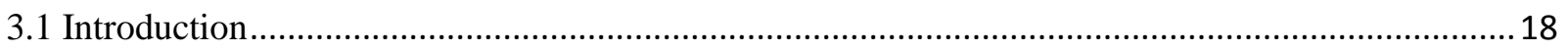

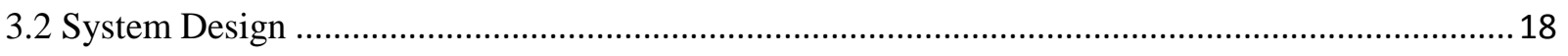

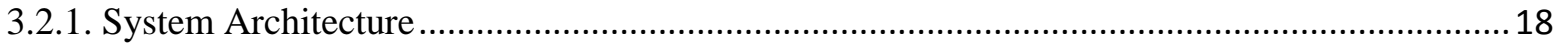

3.2.2 UML Sequence Diagrams for Administrator and Regular User Tasks...................................21

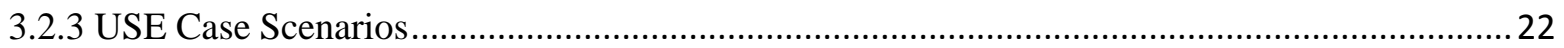

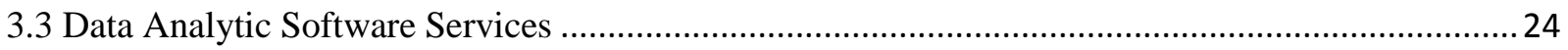

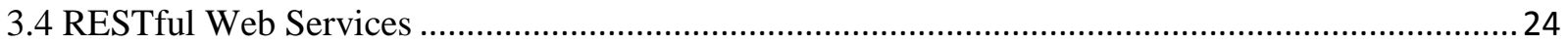

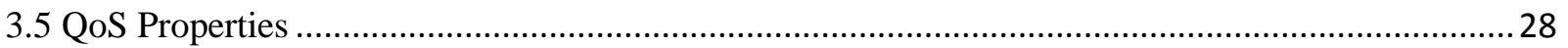




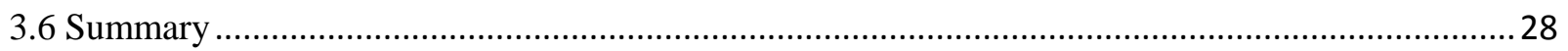

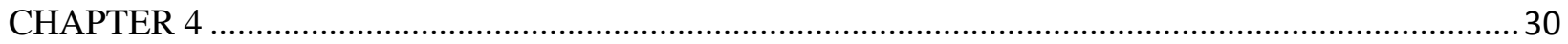

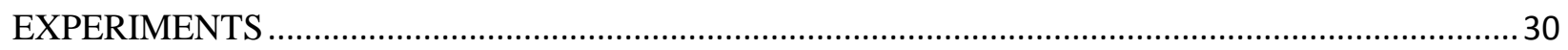

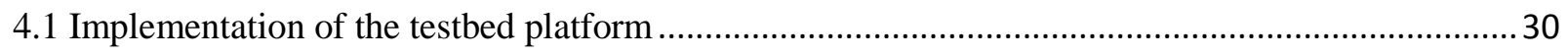

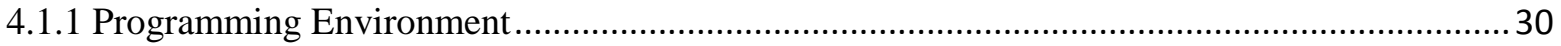

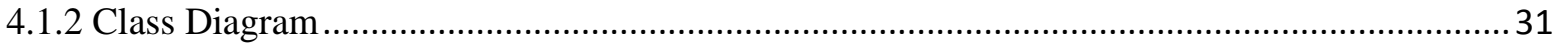

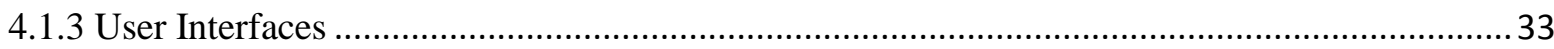

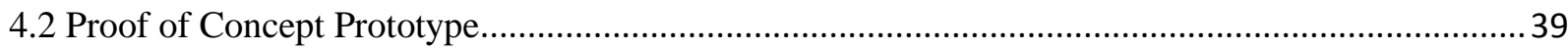

4.2.1 Data Mining Packages ................................................................................................... 40

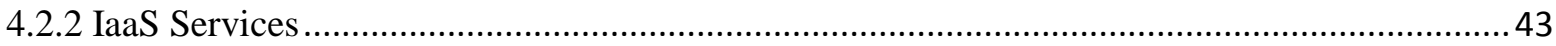

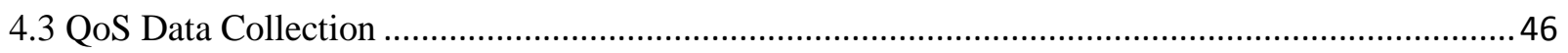

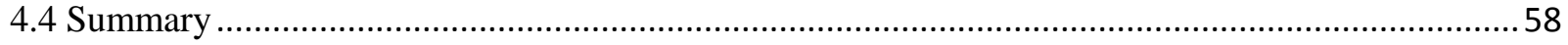

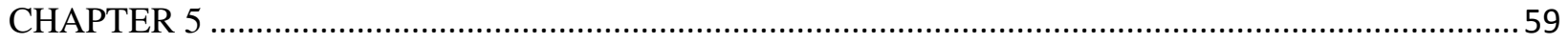

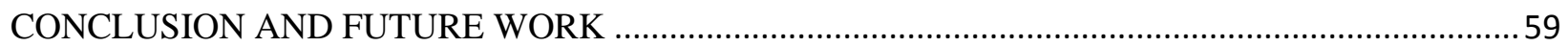

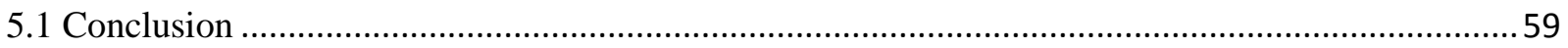

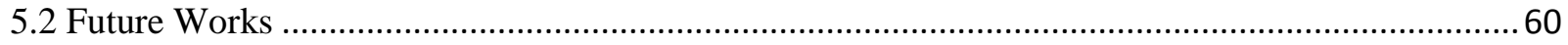

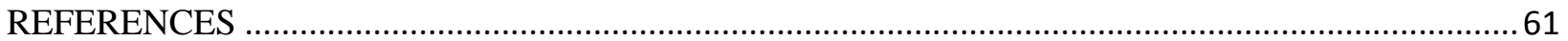




\section{LIST OF TABLES}

Table 4.1: Hardware configuration ..........................................................................................

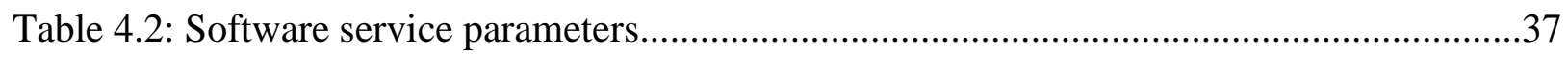

Table 4.3: List of Implemented Weka Algorithms...................................................................41

Table 4.4: List of Implemented R algorithms.........................................................................42

Table 4.5: List of Implemented Apache Commons Mathematics machine learning algorithms..43

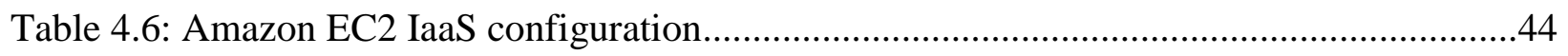

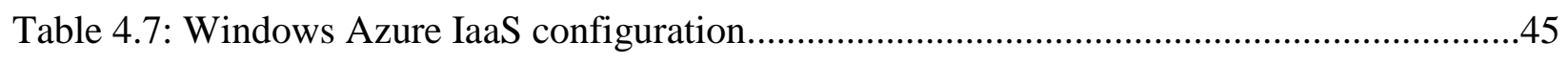

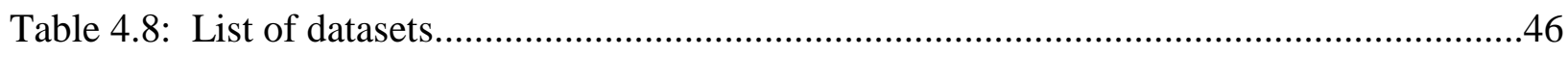

Table 4.9: QoS values for one dataset (Balance Scale), one IaaS (Azure-1) and all services.......49

Table 4.10: QoS values for one dataset (Lung Cancer), one IaaS (Azure-1) and all services.......50

Table 4.11: Average QoS values on one IaaS (Azure-1) for all dataset........................................51

Table 4.12: Average QoS values of one SaaS (Weka FarthestFirst Clustering) for all dataset.....52

Table 4.13: Average QoS values for one dataset (Lung Cancer) on all IaaS................................53

Table 4.14: Average QoS values of one SaaS (Weka SimpleKMeans Clustering) on all IaaS.....54

Table 4.15: Average QoS values of all SaaS for one dataset (Lung Cancer).................................55

Table 4.16: Average QoS values of all SaaS on one IaaS (Azure-1)..........................................57 


\section{LIST OF FIGURES}

Figure 1.1: Basic service-oriented architecture ....................................................

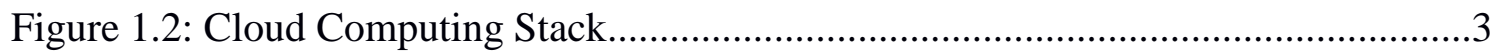

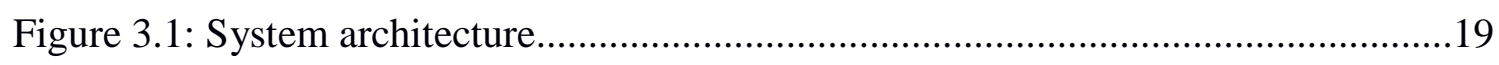

Figure 3.2: UML sequence diagram for service invocation.......................................21

Figure 3.3: UML sequence diagram for administrator tasks.....................................22

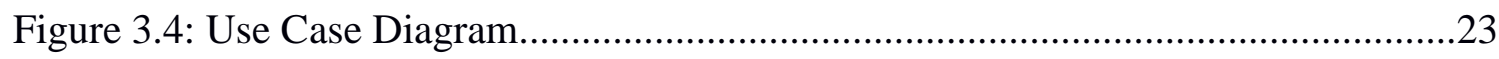

Figure 3.5: Simple RESTful web service request and response .................................26

Figure 3.6: Simple SOAP-based web service request and response.............................27

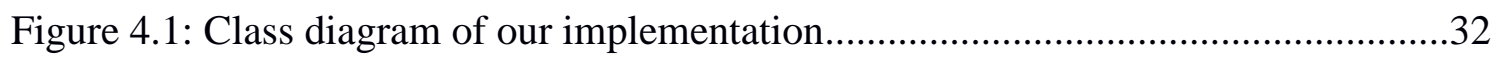

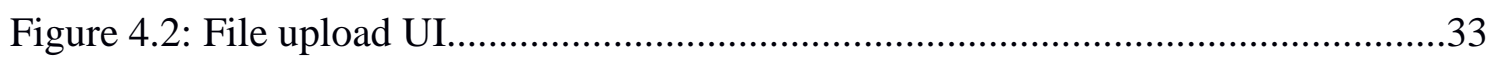

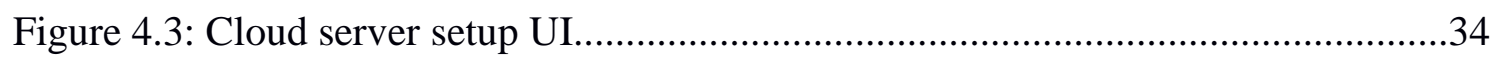

Figure 4.4: Web service publish or registration UI................................................35

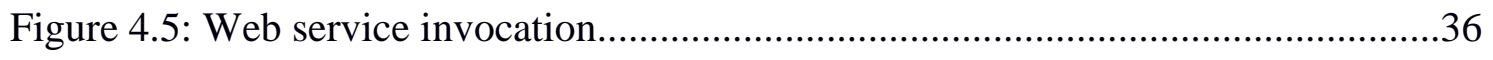

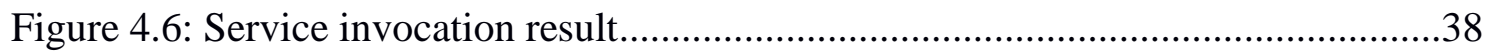

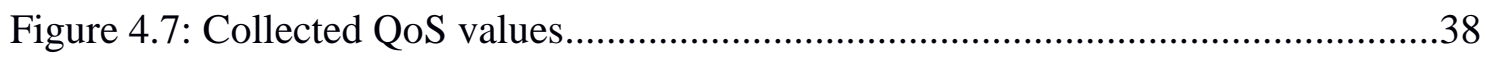




\section{LIST OF ACRONYMS}

AHP: Analytic Hierarchical Process

AMI: Amazon Machine Image

API: Application Programming Interface

AWS: Amazon Web Services

EC2: Elastic Compute Cloud

HTML: HyperText Markup Language

IaaS: Infrastructure as a Service

JSON: JavaScript Object Notation

MVC: Model View Controller

OASIS: Organization for the Advancement of Structured Information Standards

PaaS: Platform as a Service

QoS: Quality of Service

REST: Representational State Transfer

SOA: Service-Oriented Architecture

SOAP: Simple Object Access Protocol

SaaS: Software as a Service

URI: Uniform Resource Identifier 
URL: Uniform Resource Locator

WSDL: Web Service Description Language

XML: Extensible Markup Language

WEKA: Waikato Environment for Knowledge Analysis 


\section{CHAPTER 1 \\ INTRODUCTION}

\subsection{Background}

According to Wikipedia [1], service refers to "a set of related software functionalities that can be reused for different purposes, together with the policies that should control its usage". In another definition according to OASIS [2], service is "a mechanism to enable access to one or more capabilities, where the access is provided using a prescribed interface and is exercised consistent with constraints and policies which are specified in the service description".

Service-Oriented Architecture is a kind of software architecture that contains a collection of services where these services communicate with each other. Sometimes this communication could be simple, such as simple data passing, and sometimes it could involve two or more services communicating with each other to do some activity.

Figure 1.1 illustrates the basic service-oriented architecture. Service providers publish their developed services in a service registry. Service registry is a repository for all published web services. Service consumer looks for the service in the service registry and then invokes the service. The communication happens in such a way that both service provider and service consumer can understand. The actual communication depends on the implementation of the web service. Two popular communication technologies called Simple Object Access Protocol (SOAP) and Representational State Transfer (REST) are used to communicate between two services. 


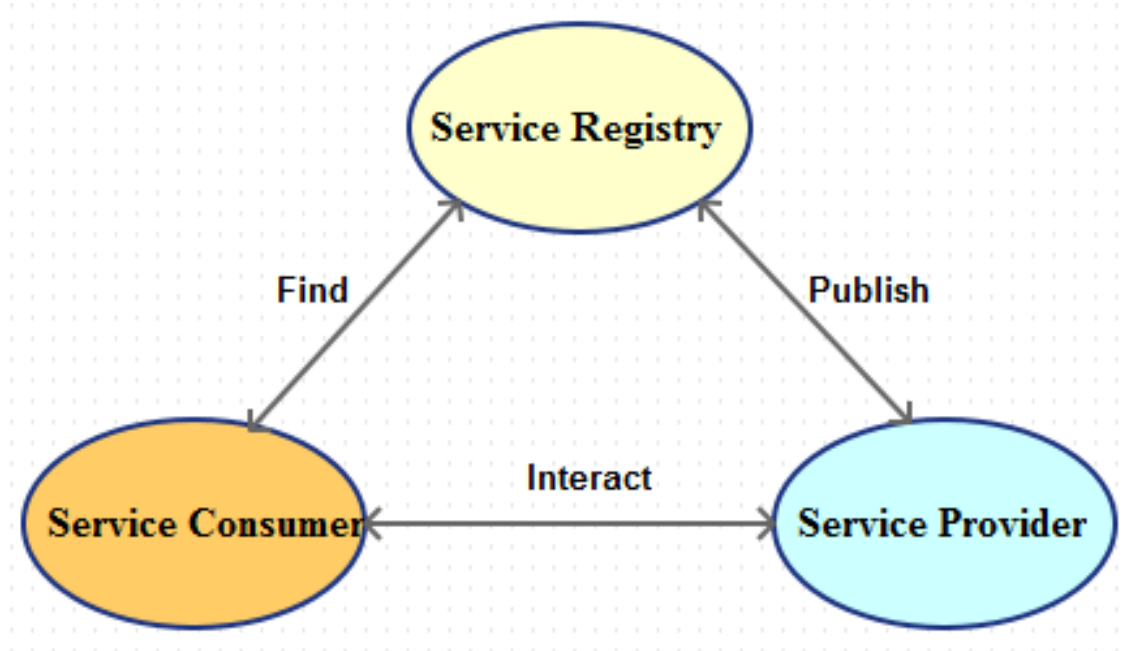

Figure 1.1: Basic service-oriented architecture

In every business, organizations need to invest time and budget to scale up their IT infrastructure such as hardware, software and services. With on premise IT infrastructure, scaling process can be slow and organizations are frequently unable to achieve optimal utilizations of IT infrastructure. Cloud computing consists of highly optimized virtualized data centers. They provide various software, hardware and information resources on demand. In another definition, Cloud computing is a distributed computing model where different kinds of resources are available in an on-demand pay-as-you-go model [3]. Organizations can simply connect to the cloud over the internet and use the available resources whatever and whenever they need. Cloud computing consists of three main service models including IaaS (Infrastructure as a Service), PaaS (Platform as a Service and SaaS (Software as a Service) as shown in Figure 1.2. 


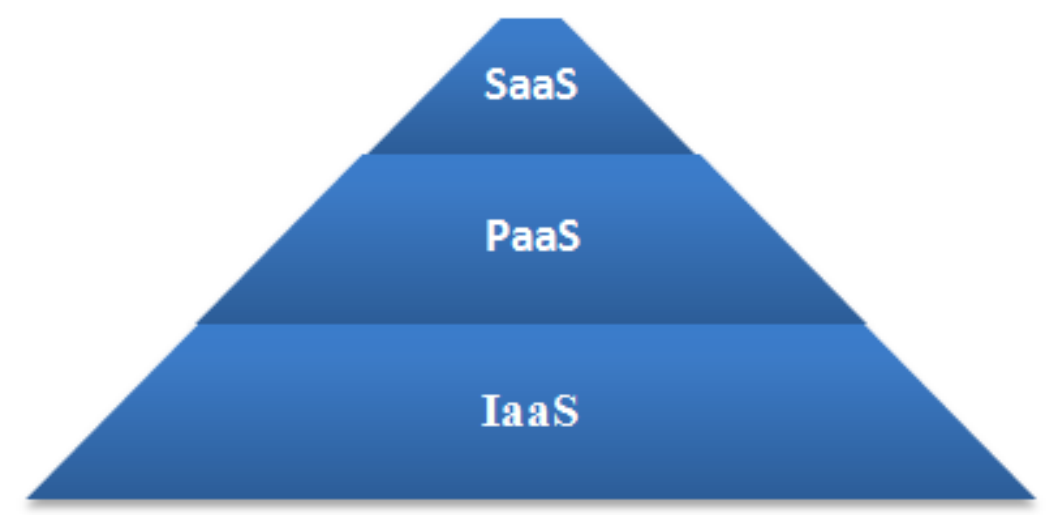

Figure 1.2: Cloud Computing Stack

IaaS is to rent cloud infrastructure from Cloud service providers such as server, storage and networking in an on-demand basis. As companies always want to invest optimal budget for their business, IaaS can be a solution for them because customers do not need to pay for hardware cost as they lease shared resources. As cloud infrastructure scales resources ondemand, it is a great opportunity for the company to implement the workload balance. PaaS is the platform containing a set of tools designed to make coding and deploying the applications easier. Cloud-based applications or software services (SaaS) run on far-away computers in the cloud that are connected to users' computers via Internet, usually via a web browser. The SaaS providers can also provide API to access their application and data for the developers to use in their own application for creating composite applications. It is pay-as-you-go, so that users don't have to invest a lot in purchasing software packages. The major architecture of SaaS is based on multi-tenant model. A single version of the application is installed for all customers considering single configuration with this model. Horizontal scaling is implemented on multiple machines to support scalability. Vertical scaling can resize the cloud server with no change of the code. 
The purpose of cloud computing is to make available resources for the cloud users, where they can access rented cloud infrastructure and software applications from anywhere and anytime. The focus of this research work is on software services hosted in the cloud environment, and we specially pay attention to data analytic software services.

As the number of analytic software services with similar functionality is increasing in the Cloud, service selection becomes a very important issue of Cloud computing, because it directly impacts the user experience in the Cloud. It is difficult for the customer to decide which services to use to meet their requirements among the vast number of services in the cloud. QoS (Quality of Service) - parameters (e.g., response time, throughput, availability, reliability, latency) play an important role in the service selection process. Sometimes users can select services based on their functionality (e.g., a clustering service), not considering their QoS values, but in most other cases functional requirements are not enough and QoS requirements should also be considered. In this work, for non-functional requirements, we mainly concentrate on the QoS-based selection of data analytic software services.

\subsection{Problem Statement}

In the last few years, researches were focused on service selection using different technologies including vector and matrix based approach [4] [5] [6], constraint programming or integer programming approach [7], multi-criteria decision making approach [8], semantic approach [9], etc.

When more services are moving to the cloud, there are also research works on cloudbased service selection. Some focus on software service selection [10] [11], and others focus on infrastructure service selection [12]. With the emergence of Big Data, analytic-as-a-service has attracted some research attention [13] [14] [15]. Researchers have started to implement analytic 
software such as data mining algorithms as services and hosted them in the cloud. When more and more this type of analytic services are hosted in the cloud, it would become a challenge for users to find the right service to process their data.

Although there are many QoS-based service selection models (either web services or cloud services) that have been proposed, to the best of our knowledge, there are very few benchmark datasets available for researchers to compare the results of their models. QWS dataset [16] is one of the most popular ones. WS-Dream [17] is another one which has been used in several papers. Both of them are providing data of web services. Most of the research works for cloud-based service selection either use web service QoS data or simulation data to do the experiment. No real cloud-based end-to-end QoS data has been collected for the research purposes in the past.

When selecting data analytic services in the cloud, there are a few unique challenges: 1) the QoS values of services could be affected by the dataset to be processed, for example, the latency on a big dataset could be longer than the one on a small dataset, the accuracy of a service could be low when there is noise in the data; 2) in a cloud environment, a software service could be hosted on different infrastructure services, and thus the end-to-end QoS values could be affected by both software service implementations as well as infrastructure service configurations; 3 ) the service selection should be based on the end-to-end QoS values, and thus there should be a monitoring service offered by the system to collect these end-to-end QoS values instead of depending on published values from individual providers. Because of these challenges, to facilitate the research on cloud-based analytic service selection, it is important to set up a broker-like testbed framework in the cloud environment. This testbed framework can be used to publish services and software services will be hosted on different infrastructure services. 
The hosted services can be invoked with end-to-end QoS values being monitored, and both dataset properties and QoS values from different services can be collected.

Because of these unique challenges for cloud-based data analytic service selection, and considering that there is no proper benchmark dataset available for the research in this area, it is the purpose of this thesis work to propose and implement a cloud-based testbed. Using it we can collect end-to-end QoS values considering the effects from software services, infrastructure services as well as the input datasets. With the collected QoS dataset, researchers can evaluate and compare the performances of their selection algorithms.

\subsection{Objectives}

There are two major objectives of the work. First, we want to design a cloud-based framework to build testbed for collecting end-to-end QoS values of data analytic services. Second, we want to implement a proof of concept prototype system. In this system, data mining services are created as representative analytic services, infrastructure services with different configurations and in different locations are selected from two different providers (Amazon EC2 and Windows Azure). A client tool is developed to communicate with our data mining services hosted on different infrastructure services, invocation requests of applying these services on some real datasets are sent and end-to-end QoS values are collected from a monitoring engine.

\subsection{Proposed Approach}

The proposed framework is similar to a cloud marketplace, in which different providers can publish their services and users can select different services. However because our focus is on collecting QoS data, we are not going to implement a full cloud marketplace with all the required functionalities. We choose 3 major components to be included in our framework: 1) 
publishing component for providers to publish their services, 2) invoking and monitoring component for users to invoke a service and for the system to record the end-to-end QoS values, and 3) simulation component to run a batch invocation job so that all the selected services will be invoked one by one on a given dataset and all the QoS data are collected.

In our proof-of-concept prototype, we chose three package providers for the data mining algorithms to be developed for our software services. We chose open source data mining packages from WEKA [18], R [19] and Apache Commons Math Machine Learning [20]. We developed 22 software services using 3 categories of data mining algorithms including Clustering, Classifier, and Association rule mining. For the service implementation, we used the RESTful web service framework to develop the software service. We hosted our developed software services in two different cloud infrastructures including Amazon EC2 and Windows Azure instances in different locations with different configurations. For the invocation of our services, we developed a client application which allows users to upload their own files, invoke data mining services to process data, and get the QoS values recorded. The collected QoS values are stored into a repository and these QoS values could serve as benchmark data for other researchers to compare their selection models or algorithms.

\subsection{Organization of the Thesis}

The rest of the thesis is organized as follows:

Chapter 2 reviews and analyzes the existing research work in the field of web service selection, cloud service selection, and cloud marketplace and brokers. It also reviews the existing research efforts that are closely related to our work such as QoS-based service selection, data analytic services, data mining as a service, etc. 
Chapter 3 gives the details of our proposed methodology, including the architecture model, functions of different components and the system workflow. We also discuss about the RESTful web service framework for building our software services and how to set up infrastructure services from different cloud service providers to host the software services. Chapter 3 also talks about different QoS properties that we have considered in this thesis work.

Chapter 4 explains how we implement the proposed testbed framework, shows some key interfaces of our system, describes the details of our proof-of-concept prototype implementation, including data mining software services and infrastructure services and their configurations, discusses the QoS data collection process, and, finally, reports the statistical information of a collected sample dataset, which showcases the impact of dataset, infrastructure configuration and location, as well as software service algorithms on the QoS values of services.

Finally, in Chapter 5, we conclude our thesis with a summary of results and analysis. We also talk about what could be the possible future enhancement of our work. 


\section{CHAPTER 2}

\section{LITERATURE REVIEW}

\subsection{Introduction}

As our ultimate goal is to provide a platform for the study on QoS-based service selection problem in the cloud environment for other researchers, we would like to discuss first some existing works on QoS-based service selection. After that, we would like to review papers on cloud marketplaces, cloud brokers, cloud service selection and data analytic services.

\subsection{QoS Based Web Service Selection}

Since many software services with similar functionalities are published by various providers, it is a big concern for the end-users to choose software services that best meet their requirements. The non-functional attributes of any software services such as response time, latency, availability, reliability, and throughput, can play an important role for the service selection process. Different types of service selection approaches are introduced in various papers.

In [21], Mixed Integer Programming (MIP) is used for solving the problem of service selection and match-making for linear constraints, while Constraint Programming (CP) is used for non-linear constraints. The experiment shows that MIP performs better than CP while

considering linear constraints. So the match-making procedure can be completed using $\mathrm{CP}$ or MIP depending upon whether it is non-linear constraints or linear constraints.

In [22], the user preferences are defined using utility functions, a probabilistic trust model is used to get quality distributions of providers, and then the services which could maximize the expected utility values are selected. 
In [23], the QoS manager acts as an agent for service providers and clients to do publishing and finding web service operations. QoSDB has been used to store the details of QoS of a web service and QoS values such as response time, throughput, reliability, availability and cost are optimized and the ranked values are stored in QoSDB. To find the best web services, the user has to specify the functional details of the service and its QoS values which are required to find the list of web services from the repository.

Analytic Hierarchical Process (AHP) is a popular Multi-Criteria Decision Making (MCDM) technique that solves problems by modeling QoS characteristics in the form of a hierarchy [24]. For the hierarchy, the multiple criteria are divided into sub-criteria and could be further divided and thus establishing different levels of criteria. The relative ranking of the various options is calculated based on the priorities associated with the criteria and their sub criteria within each level and finally the overall rank of each alternative can be determined.

As we focus on QoS-based attributes, there are a few commonly used QoS attributes [5] [16] for assessing the performance of a service. Reliability indicates how many error messages have been generated compared to the total number of messages. Latency is considered as the time taken for the request to be processed. Response Time is the time it takes to send a service request and receive the response. Throughput represents the maximum number of invocations that can be managed in a certain time period. Availability is defined as the percentage of time the service and server are available.

\subsection{Cloud Service Selection}

In the service computing paradigm, web services are referring to software services hosted and provisioned by software providers through the Internet. In the cloud computing paradigm, there are many types of cloud services. Besides software services, there are also infrastructure 
services, platform services, database services, storage services, etc. With a variety of services, the research on cloud service selection has some new challenges. Most of the research work nowadays is focused on either software services or infrastructure services, and various methodologies have been used. A fuzzy simple weighting system has been proposed in [25] to normalize and aggregate two types of attributes of a cloud service. They consider subjective assessment from cloud consumers and objective performance assessment from a trusted third party. Then they apply fuzzy weighting system on the subjective and objective information for normalization and aggregation, so that some specific performance aspects of a cloud service can be considered accordingly for potential cloud users.

MSSOptimiser (Multi-tenant SaaS Optimiser) approach has been introduced in [7], where functional specification and QoS requirements could be input of the proposed model. Integer Programming could be used to determine the optimal services. For large-scale scenarios, SaaS optimization problem is expensive, but MSSOptimiser provides greedy heuristic to provide an optimal solution efficiently.

In [26] [27], a hybrid fuzzy framework for cloud service selection has been proposed using three approaches: 1) fuzzy ontology based approach for functional matching; 2) fuzzy AHP for weighting; and 3) fuzzy TOPSIS approach for service ranking. Bi-partite graph has been used to find functional matching results. In their model, they focus on customer's fuzzy perception of QoS. To deliver more certain information into the ranking algorithm, they convert linguistic terms of customer's perception into precise numbers.

To solve the problem of cloud service selection based on multiple criteria, a rigorous mathematical model has been used but their approach is only effective to select amongst service offerings that are similar in specification but different in performance. 
In [28], the proposed methodology considers QoS parameters as the decisive factors for selecting appropriate cloud service. The model called Service selection for Data-centric cloud application (SSADCA) based on normalization mechanism is capable of accommodating any number of QoS parameters. The user is also able to accommodate any order of preferences of the QoS parameters.

AHP based methodology is used in [29]. The proposed methodology is two-fold: 1) consumer-centered service selection considering user's preference in multiple context (single user and multiple users); 2) making reasonable cloud service selection based on Cloud Service Decision-Making (CSDM). The proposed method is capable of automatically selecting cloud infrastructures. They consider a couple of criteria for their selection algorithm including deployment cost, location of cloud infrastructures, application clients, related applications, and communication among application components. They also introduced stepwise application placement algorithm for addressing scalability issue when the number of data centers and applications is large.

One of the main challenges for SaaS providers is to select best IaaS providers. To achieve this goal, SaaS provider needs to map user's requirements in QoS specification into the IaaS layer [30]. The work in [30] develops a set of mapping rules and a new way to calculate QoS values in a cloud environment using AHP method. QoS weights for service selection and ranking process are generated using AHP method based on QoS specification model.

\subsection{Cloud Marketplace}

Cloud marketplace is online storefront that is managed by cloud service providers or third parties. In a cloud marketplace, SaaS providers publish their software services and customers may subscribe to that software. Cloud service providers provide facilities to their subscribers to 
use their already built-in cloud applications and approved applications from the third party. This approval of third party applications not only gives opportunity to the customers to use more applications, but also fills up the gaps for demanding more applications by their subscribers.

In [31], the market negotiation is the main focus of the paper, but they also attempt to define all important entities of the proposed marketplace such as sellers, buyers, intermediaries, etc. Market negotiation is the main functionality of the proposed marketplace that happens between the sellers and buyers who subscribe in the marketplace. The automation of the negotiation mechanism, inclusion of multiple attributes, and being a short term commitment are some of the characteristics.

The pricing model for IaaS resources is the main objective of the proposed model in [32]. The model is supposed to bring both a long term pricing contract for the benefit of IaaS providers and a pricing scheme that is based on users' actual jobs. Some other features of the proposed model are: 1) economic benefits, 2) ability to provide a broader view of the user's usage of cloud resources, and 3) better predictability compared to the traditional pricing models adopted by current cloud providers.

In [33], the main idea of the proposed marketplace system is to create interfaces for all cloud parties (e.g. users and service providers) to interact for business functions, and make the work flow easy to implement. The proposed architecture isolates the interface component from the core system logics. As a result, the marketplace could easily and quickly engage in the service provisioning process. It is designed to be flexible and used for all service functionalities, and by any providers and private developers. The structure of the proposed marketplace basically represents each entity as a logic block and each communication link as an interface (API).

The problem of service selection in a cloud marketplace is addressed in [34]. Cloud 
marketplace should be able to support service composition, spanning different cloud layers and providing tools for providers to use existing products. The proposed solution takes into consideration the different layers of cloud entities along with the service business aspect specifically the service pricing. In their design view, the marketplace plays the role of a broker between the different service providers and their consumers. In their concept of multiple cloud layers, the business aspect and pricing are the main criteria for service selection mechanism.

The VMware vision of cloud marketplace has been discussed in [35]. They emphasized that marketplace should be open especially for general purposes, to all vendors and developers to submit their offers. The deployment procedure must be easy in the cloud environment. The paper focuses on IaaS providers and it deals with different types of consumers in a way that it helps them avoid much of expenses in acquiring actual resources.

In [36], a new model for cloud marketplace has been proposed where IaaS providers can work collaboratively to provision services. Finding relationships between providers who can form a single combined service offer and end users are the main goal of this paper. This strategy has different advantages such as the service cost can be reduced for the customer, the negotiation time is reduced and conflicts between services' requirements are minimized while maintaining QoS requirements for customers.

\subsection{Cloud Broker}

A cloud broker is simply a third-party individual or organization that acts as an intermediary between customers and sellers of the service in a cloud environment. Cloud broker works with customers to help them understand work processes, budgeting, data management systems and saves purchase time by providing information to customers. There are three types of

cloud brokers [37] including cloud aggregators, cloud enablers and cloud agents. A Cloud 
aggregator provides the customer information about Application Programming Interface and User interface complexity. A Cloud agent is a software application that helps to distribute the works among different cloud service providers. A Cloud enabler must provide the customer some additional services such as encryption, transfer of customer data to the cloud and help them understand the data lifecycle management.

In [38], a brokerage-based architecture is proposed, where cloud broker is responsible for service selection. They design a unique indexing technique for managing the information of a large number of cloud service providers and develop an efficient algorithm that ranks potential service providers and aggregates them if necessary.

A cloud broker service called STRATOS is proposed in [39], which is an initial version of a broker service layer. This initial step could be used for cross-cloud resource provisioning. The broker service also facilitates application topology construction and runtime modification, based on deployer's objectives. They also emphasize the importance of mechanisms to compare and normalize offerings from multiple providers.

The self-adaptation and self-management techniques can improve service-oriented systems because they can tackle the complexity of a complex system and their environments by themselves. In [40], the authors designed a broker system called MOSES (MOdel-based SELFadaptation of SOA systems) which provides runtime QoS-driven adaptation of SOA applications. They developed two versions of MOSES. In version 1, they got some issues including network overhead and storage access problems etc. In version 2, they used OpenESB (Open Source Enterprise Service Bus) that replaced Tomcat with Glassfish and also maintained the same functionalities of MOSES 1 including standard way for communication and easy integration of new components. 


\subsection{Data Mining Services and Data Analytic Services}

Data mining and data analytic services have attracted more and more attention from different sectors including academia, industry, and government. There is no proper benchmark tool for data analytic services to collect end-to-end QoS values in the cloud. In [41], the authors introduced cloud-based big data mining and analysis service platform by integrating $\mathrm{R}$ in their model. In the proposed architecture, there are four layers: infrastructure layer, virtualization layer, dataset processing layer and services layer. Apache CloudStack was installed, configured and deployed to construct virtual machine clusters to manage infrastructure resources. In data processing layer, R language runtime environment and RHadoop environment were setup. There are four aspects to be considered in services layer including computing mode selection, cloud services, data mining workflow and user interface. They applied K-Means algorithm as service in their model.

In [42], a comprehensive and collaborative workspace for data professionals and analysts as cloud-based data mining platform, called DMaaS (Data Mining as a Service) is proposed. Their data processing engine is based on Hadoop and they deployed their services considering some of the data mining algorithms from Apache Commons Math Machine Learning [20]. The end-users can access their services from their own browsers easily.

Analytics as a Service refers to the provision of analytics software and operations through Web-delivered technologies. Data Analytics as a Service (DAaaS) is an extensible analytical platform in cloud environment where various tools for data analytics are available and can be configured by the user to process and analyze huge quantities of data. Many of data analytics services are based on Machine Learning concepts. The DAaaS platform has the ability to support the complete lifecycle of its analytics capabilities. There are some challenges of analytics 
services in the cloud such as information lifecycle management, security of the data, privacy, real-time analysis and data volume. In [47], the authors introduced an AaaS tool that can be used to accomplish the extraction and organization of terms and topics from unstructured data sources including NoSQL, flat and PDF files. They proposed, designed and implemented the framework.

A collaborative big data analytics platform for big data as a service was proposed in [48]. This paper described big data analytics platform to manage big data and developed analytics algorithms and services which could collaborate with data owners, data scientists and service developers on the web.

\subsection{Discussion}

Since our objective in this work is to provide end-to-end QoS values of a service in the cloud and these QoS values could be used for service selection algorithm, in this chapter, we first reviewed papers about QoS based service selection. We created some data mining services as representative data analytic services and hosted them in different cloud infrastructure. Thus we also reviewed papers on data analytic services, data mining and cloud marketplace. For data analytic services, QoS values are depending on dataset features and they are different for different datasets. However, based on our review, most of cloud marketplaces and brokers focus on different techniques including indexing techniques, AHP method and QoS weights for service selection, etc., and none of them tried to collect the end-to-end QoS values which are essential for service selection. From the above discussion, we see there is no proper benchmarking tools for collecting end-to-end QoS values and these QoS values are important factors for service selection. 


\section{CHAPTER 3}

\section{METHODOLOGY}

\subsection{Introduction}

In this thesis work, we want to create a framework, in which new analytic services can be published with related information saved in a repository, users can search and invoke services, and the QoS values of these invoked services can be monitored and recorded. Since we focus on data analytic services, QoS data is associated with not only the service but also the dataset being processed. As a proof of concept, we have applied this framework to create a testbed, in which we have selected a few types of algorithms (i.e. clustering, classification, association rule mining), each type with a few different algorithms implemented, from different data mining software packages (i.e. Weka, R, Apache Commons Math Machine Learning), and we have implemented all of them as software services using RESTful web service framework, hosted and delivered them through different cloud infrastructure services (i.e. Windows Azure, Amazon EC2) with different configurations, and finally invoked them with different datasets and recorded their QoS values. The generated QoS data collection can be used for research works on QoSbased analytic service selection.

\subsection{System Design}

\subsubsection{System Architecture}

Figure 3.1 shows the architecture model of our system including different components. 


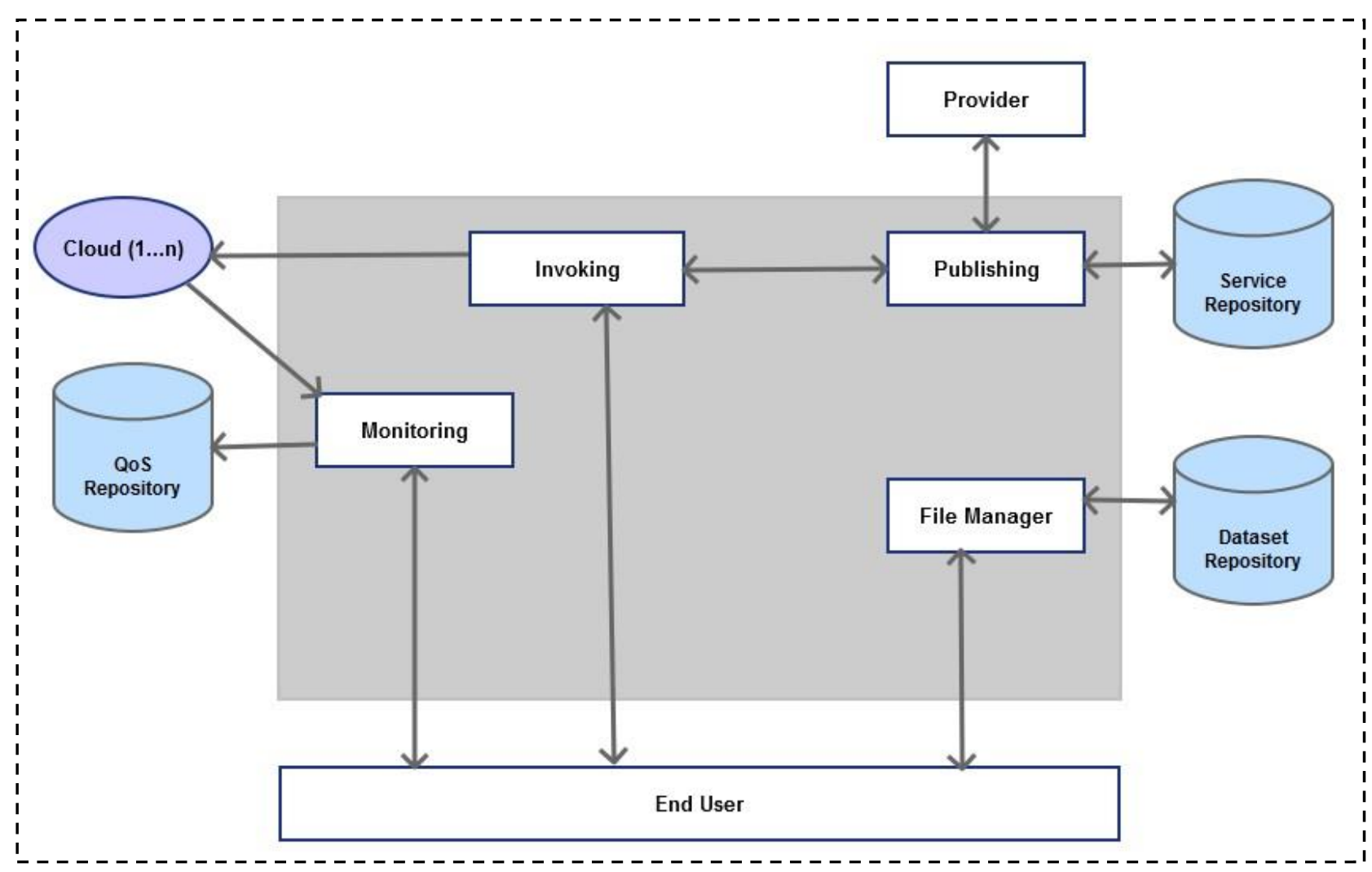

Figure 3.1: System architecture

There are 4 main components including File Manager, Publishing, Invoking and Monitoring. In our model, we have two types of users including administrators and end users. The end user is only able to upload dataset and invoke software services on the dataset. The admin user is able to do whatever end user can do and on top of that, admin user can publish software service, introduce new cloud servers, add new software services and create new users.

File Manager Component is responsible for handling user's file uploading and associating the uploaded file with the corresponding cloud server. When the end users upload their own dataset, they need to choose their desired cloud server to upload to. File Manager Component has a software service, responsible for uploading the file to the corresponding cloud server. Dataset repository keeps track of the uploaded file for that user when invoking software services. If the 
dataset is uploaded successfully, it will be stored in Dataset Repository to be used for service invocation.

Publishing component implements two main tasks i.e. Service Lookup and Service Registry. All of our software services are published in Service Registry. There is an internal communication between Service Lookup and Service Registry to avoid duplicate service publishing. Admin and providers can publish software services and end users will invoke these software services. Whenever a new software service is going to be published, Service Lookup will check whether it is already published on that cloud server. The name of the software service must be unique but provider or admin can publish same software service in different names. When any new software service is published in Service Registry, service invocation URL (Uniform Resource Locator) will be automatically generated. After a software service is published, it will be stored into Service Repository.

The main task of invoking component is the invoking of the software service. Before invoking software services, end users need to choose a data-set populating from the File Manager Component, and a cloud server and a software service populating from the Publishing Component. After the invocation, this component will return the control to the monitoring component.

After invoking a software service, our model will monitor and record values of QoS properties such as Latency, Availability, Reliability, Response Time and Throughput. These QoS values will be stored into QoS Repository and will be displayed in a browser to the end user. Later on, researchers can use this recorded QoS values for service selection. 


\subsubsection{UML Sequence Diagrams for Administrator and Regular User Tasks}

Figure 3.2 shows the UML sequence diagram of the service invocation process in our model for a regular user.

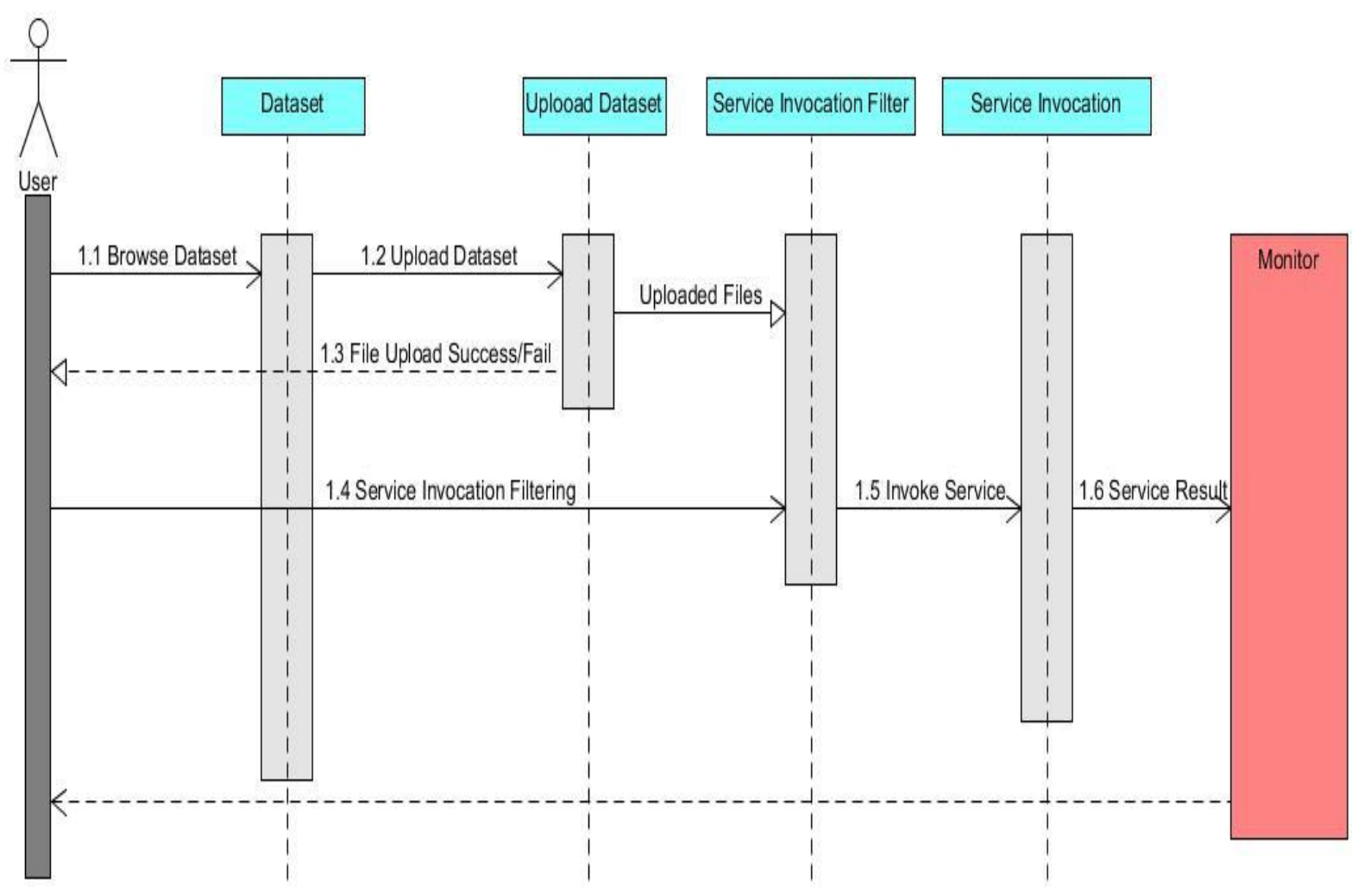

Figure 3.2: UML sequence diagram for service invocation

According to the diagram, an end user first needs to upload the dataset to the cloud server. After uploading the dataset, the end user chooses a cloud server, a software service and the parameters associated with the software service as indicated in Service Invocation Filter. After the software service is invoked, the monitored QoS values are saved and they could also be 
displayed to the end user. Software service publishing, cloud server setup and user creation are not included in this diagram. These three options are controlled by admin or provider.

Figure 3.3 shows the UML sequence diagram for administrator tasks.

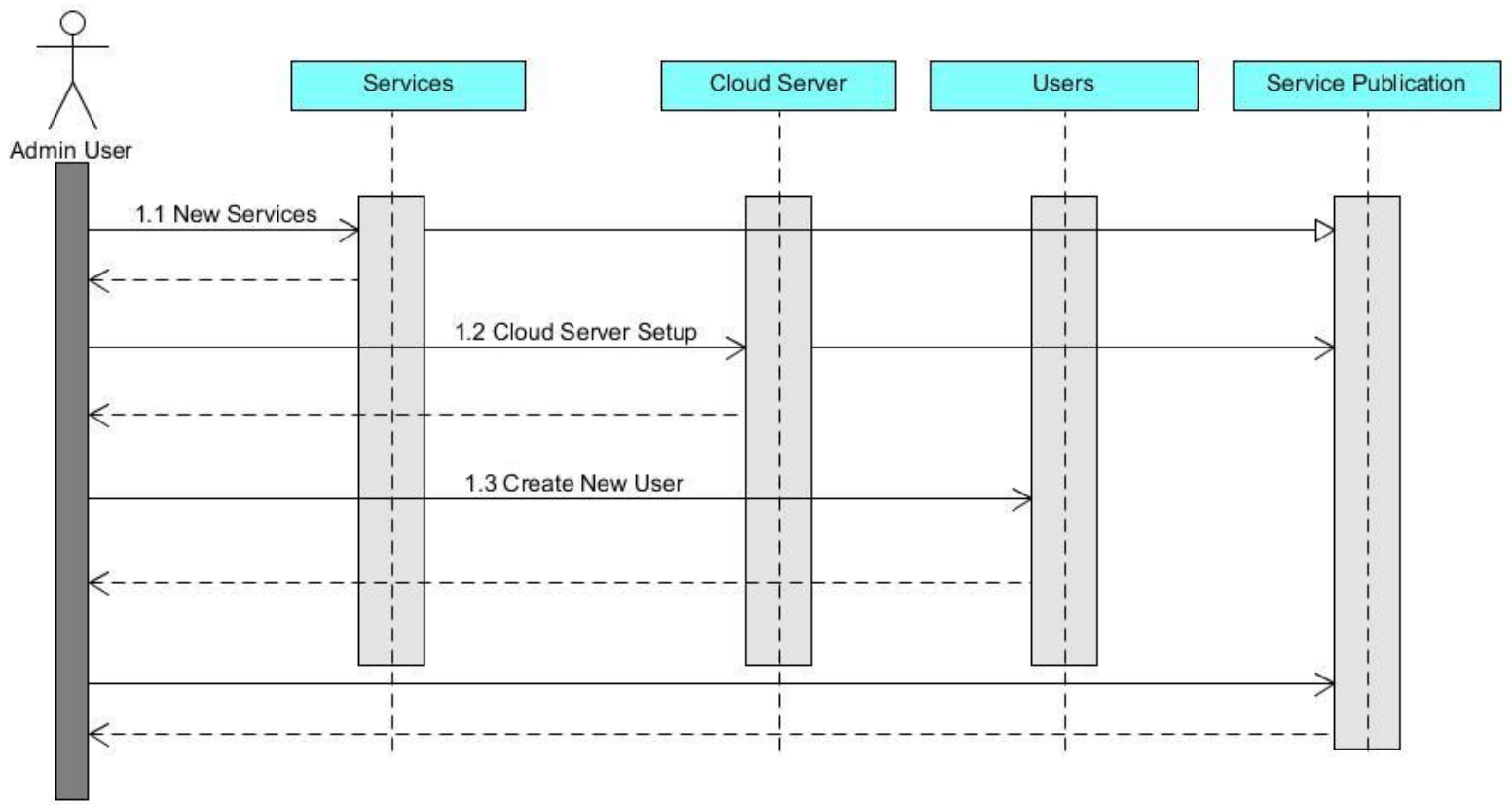

Figure 3.3: UML sequence diagram for administrator tasks

According to the above figure, there are some tasks that are not available for end user. Introducing new service, setting up cloud servers, creating new users and service publications can only be done by admin user. Besides these tasks, admin can also do tasks of regular users such as the one shown in figure 3.2.

\subsubsection{USE Case Scenarios}

Figure 3.4 shows all the use cases of our system. 


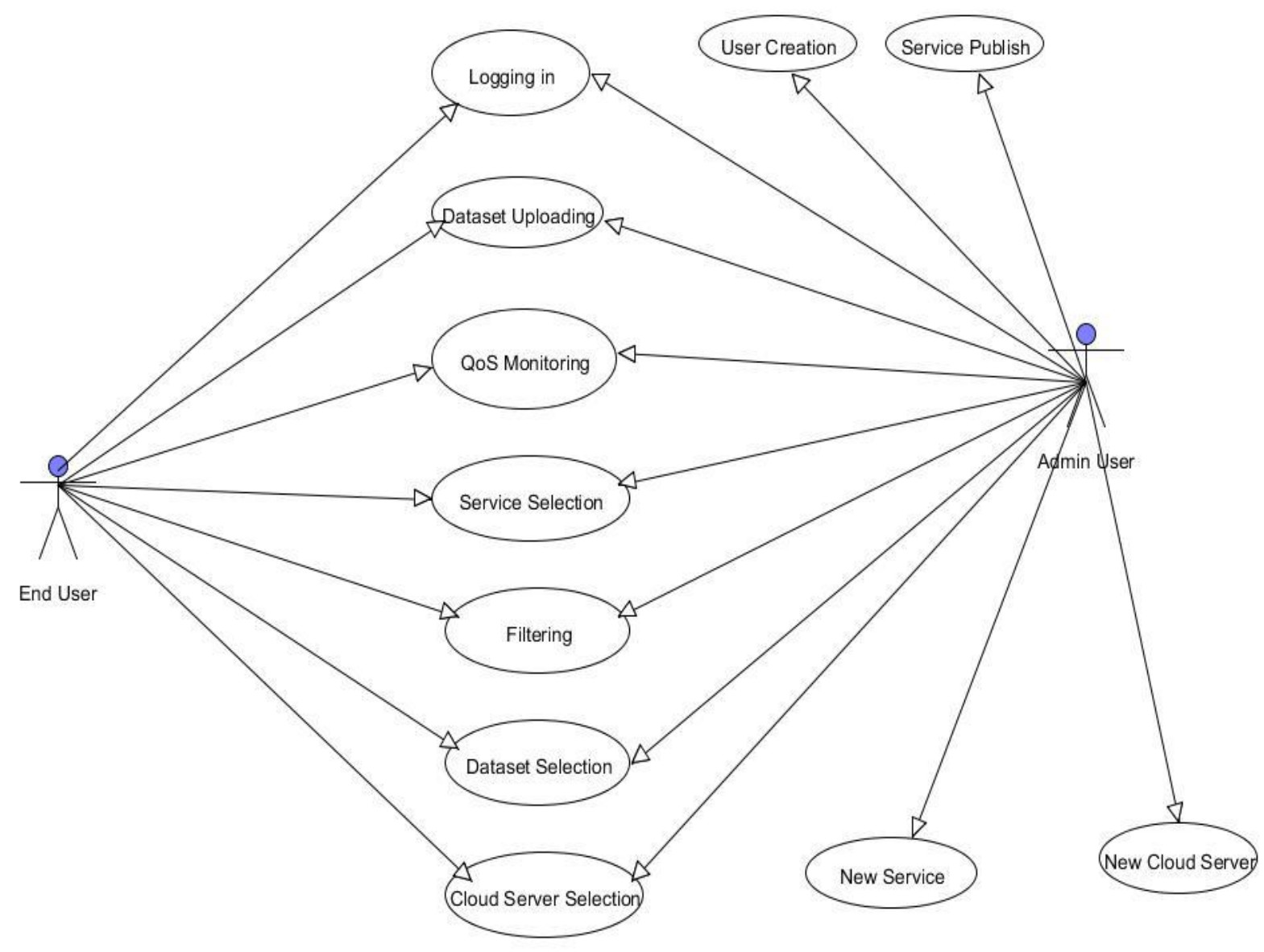

Figure 3.4: Use Case Diagram

There are two types of users in our model, i.e. administrator users or providers whose job is to configure the cloud server, publish the software service and create new users, and end users who can invoke the published services using their own datasets. According to our model, before invoking software services by end users, admin user needs to create new services, setup the cloud server and publish the software services. The users can login into the system via Logging in use case and dataset is uploaded by Dataset uploading use case. Before a software service is invoked, users need to select services in Service selection case and applying extra parameters to 
the data done in Filtering case. After selecting cloud server from Cloud server setting case, users can invoke service and the QoS value of the invocation is displayed in QoS monitoring case.

\subsection{Data Analytic Software Services}

With today's technology, more and more data are being collected. The value of the big data is not on its sheer volume. Instead, it lies on the user behavior or the hidden patterns we can learn from the data. Because of this reason, companies start to look for data mining or machine learning software to analyze the data they have saved. Many cloud providers (e.g., Amazon, Microsoft, IBM, Google) start to offer analytic software as services to their users. With all these analytic services from different providers being published and delivered in the cloud, how to select them becomes a challenge. Same as web service selection, QoS values of the services become deciding criteria to differentiate between different services. There are many factors that affect QoS values of analytic services. Similar to traditional web services, providers and their specific implementations would have impact on QoS values. On top of them, the dataset the service is going to work on, as well as the algorithm used, also have a significant impact on the QoS values, and these factors can often ignored in the current research work. In our framework, we will choose analytic services from different providers, from different categories (e.g. clustering, classification, association rule mining), and implementing different algorithms (e.g. kmeans, DBScan, hierarchical algorithm for clustering services), in order to study their impact on QoS values of the services.

\subsection{RESTful Web Services}

According to W3C definition [43], web service is "a software system designed to support interoperable machine-to-machine interaction over a network". REST [44] stands for 
Representational State Transfer. It is an architectural style for designing the system that enables clients and servers sending request and response respectively and this architectural style is approved by W3C Technical Architecture Group (TAG). REST treats everything as a resource, each resource follows the standard HTTP format and each resource has own its own name and address namely URI (Unified Resource Identifier). Each resource can have more than one representation such as HTML (HyperText Markup Language), XML (Extensible Markup Language), JSON (JavaScript Object Notation) and each representation moves over the network using HTTP protocol. RESTful web service is a great alternative to SOAP and WSDL based web service because RESTful service provides a couple of benefits to organizations such as easy integration, less memory consumption, security enablement, different output formats, better performance, scalability and support of the JSON format which is faster for parsing data. REST is being introduced in a large number of companies such as Yahoo, Facebook, Amazon, Flickr, del.ici.ous, Ebay as well as many enterprise organizations who have depreciated their existing SOAP based web services. As REST uses standard HTTP, it is much easier to use. Creating web service, client application, documentation and formatting the output in different formats are easy in REST. RESTful services communicate over HTTP using HTTP methods such as GET, POST, PUT, DELETE, etc.

Figure 3.5 shows a simple RESTful web service request and response procedure in our system. In this figure, service consumer is just sending a simple URL request to the web service which is hosted somewhere and service provider is returning response based on the request with output including the latency information. 


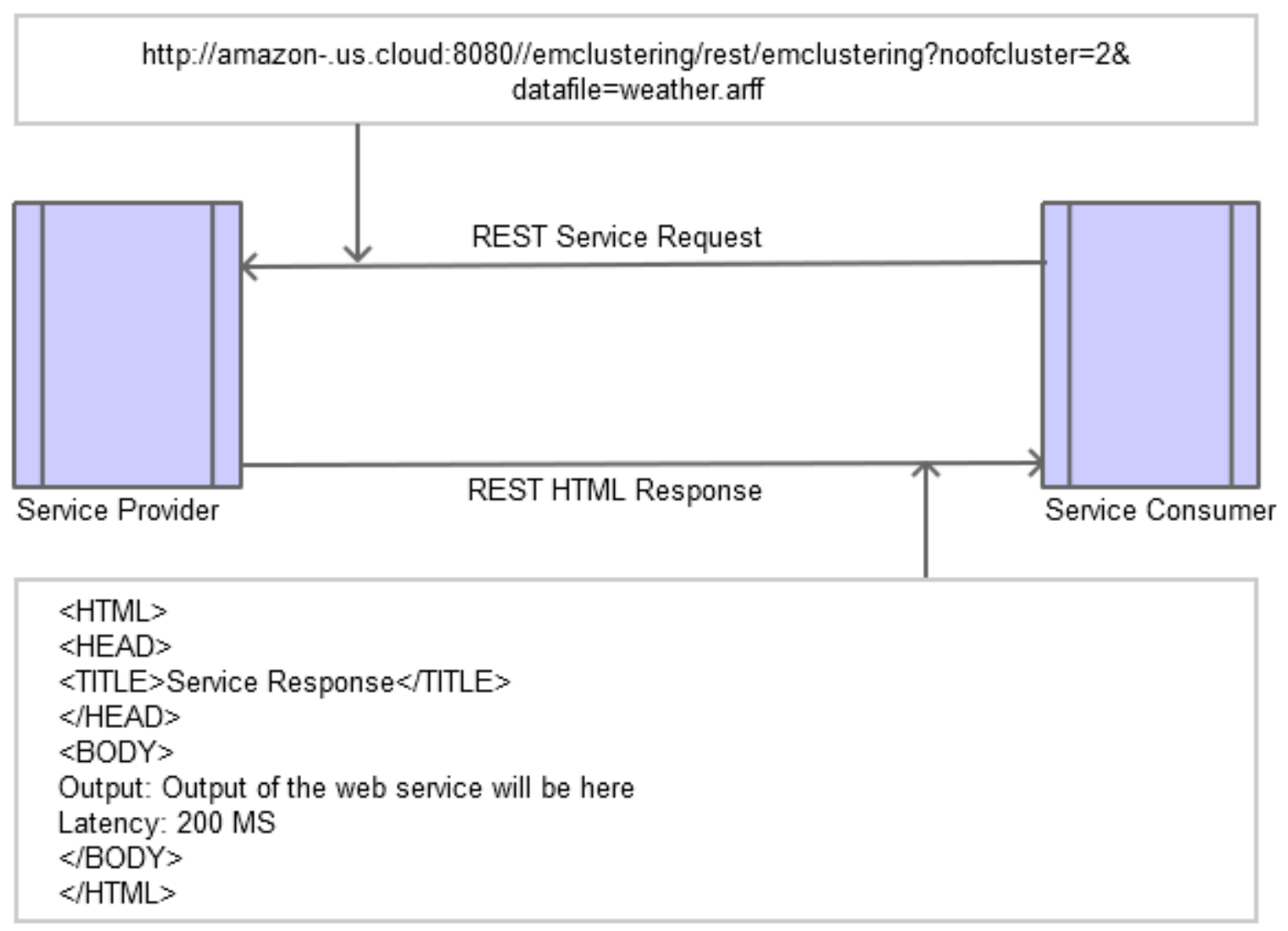

Figure 3.5: Simple RESTful web service request and response

Figure 3.6 shows how to invoke a simple SOAP based web service. From this figure, service consumer is sending a request which is in XML format and service provider is also sending response in $\mathrm{xml}$ format. 


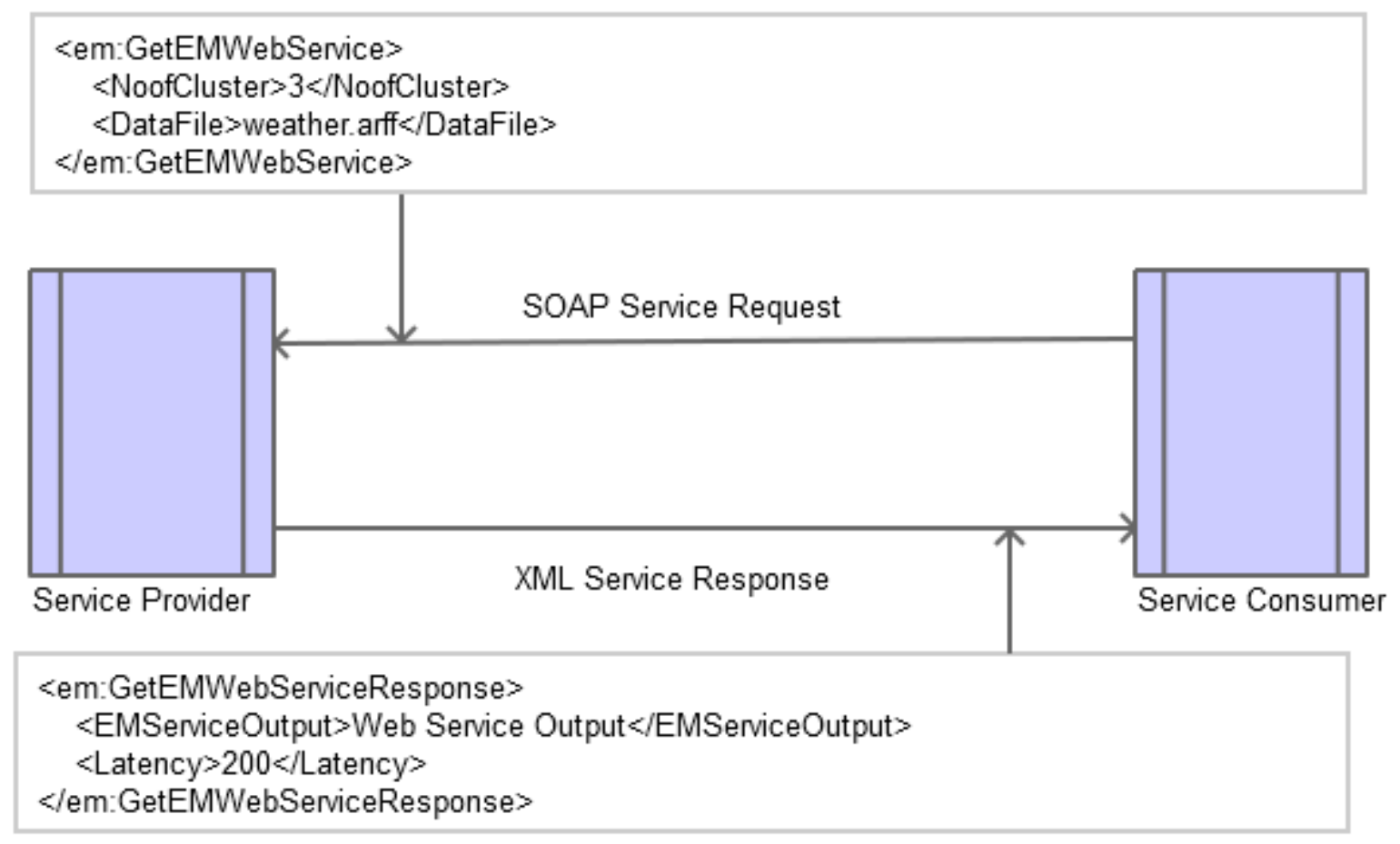

Figure 3.6: Simple SOAP-based web service request and response

These two figures show the difference between RESTful web services and SOAP based web services. Generally speaking, SOAP uses a standard communication protocol which is a specification of XML-based message exchange, whereas RESTful web service uses standard interface such as HTTP for data transmission. Compared to SOAP-based services, RESTful web services are very simple to design and implement, easy to invoke, are less memory consuming, and are completely stateless. RESTful services also provide a good caching infrastructure over HTTP Get method which can improve the performance. Considering the above benefits, we use RESTful web service in our work. 


\subsection{QoS Properties}

As our main objective is data analytic service selection in the cloud environment based on end-to-end QoS values, we need to identify QoS parameters which play an important role on service selection [16]. A service can have many non-functional properties such as:

- Response Time: Response time is the time taken to send a request and receive a response. The unit of measurement is milliseconds (ms).

- Availability: Availability is the possibility that the system is up and can be invoked by users successfully. The method of calculation is the number of successful invocations / the total number of invocations and its unit is $\%$.

- Throughput: Throughput can be measured in two ways. One way is to measure it by calculating the volume of data which is invoked at a given period of time. The other method is to measure the total number of invocations for a given period of time. The unit of measurement is invokes/sec.

- Reliability: Reliability measures the probability of a service to able to produce the correct result for a given period of time. It is measured by the ratio of the number of error messages to the total number of messages. The unit of measurement is $\%$.

- Latency: Latency is measured by time taken for a server to process a given request. The unit of measurement is milliseconds (ms).

In our work, we are considering these 5 QoS properties.

\subsection{Summary}

In this chapter we have discussed the general methodology and technologies that we used in our work. We described the high level architecture of our system explaining our four main 
components including Publishing, Invoking, Monitoring and File Manager. In our work, we only consider RESTful web services. We consider services from different providers, belonging to different categories and implementing different algorithms. We host our services in different cloud infrastructure platforms in different locations and with different configurations so that we can collect different end-to-end QoS values and investigate the impact of these different factors on the QoS values. These collected QoS values will benefit other researchers to study their QoSbased service selection approaches. 


\section{CHAPTER 4}

\section{EXPERIMENTS}

In this chapter, we will discuss about our implementation environment, proof of concept details, IaaS Configuration, our used datasets and QoS data collection. At the end, we will also analyze our collected QoS data.

\subsection{Implementation of the testbed platform}

In this work, we mainly wanted to create a testbed platform. In this platform, multiple IaaS and SaaS services can be published, and a user can choose the SaaS he/she would like to use and the IaaS he/she would like to host the SaaS, afterwards, the user can invoke the service to process the uploaded data file, and the result will be returned, and more importantly, all the QoS values for an invocation are recorded in a repository. The collected QoS values could be used for service selection research. In this section, we will talk about how we implemented the proposed testbed platform including the environment, the class diagram of our implementation and the major user interface screens.

\subsubsection{Programming Environment}

In this work, we chose Java for our system implementation. We have used Java SE (Standard Edition) Development Kit 7 and a couple of software development tools and libraries including Eclipse IDE (Integrated Development Environment) indigo, Struts2 Framework, Ant build tool, MySQL database, Apache Tomcat 7.0.35, RESTful web services, Jersey framework, Weka Explorer, R studio, Weka libraries, R programming libraries and Apache machine learning libraries etc. We chose Struts2 framework for the following reasons: 1) it follows MVC (Model 
View Controller) design patterns; 2) it communicates with the data layer; 3) it renders presentation layer etc.

Table 4.1 shows the hardware configuration used for the implementation environment and also used for running our experiment.

Table 4.1: Hardware configuration

\begin{tabular}{|ll|}
\hline Hardware & Configuration \\
\hline Manufacturer & Dell \\
\hline Processor & Intel(R) Core (TM) i7-3632QM CPU @ 2.20 GHz \\
\hline RAM & $8 \mathrm{~GB}$ \\
\hline Operating System & Windows 7 Ultimate (Service Pack 1) \\
\hline System Type & 64-bit \\
\hline
\end{tabular}

\subsubsection{Class Diagram}

Figure 4.1 shows the class diagram of our implementation. According to our model, we have three types of repositories, Dataset repository for storing datasets, Service repository and QoS repository for storing the collected QoS values.

According to our system design, Service and Cloud server are our base classes and they are used throughout the whole program. We can publish multiple services under one cloud server and that's why there is one-to-many relationship between the service and the cloud server class. Users will upload their own datasets using FileUpload class which is dependent on the cloud server class because this dependency will allow users to upload datasets to the desired cloud server. Dataset is uploaded to the cloud server by web service called UpdateSoftwareService. For publishing software services into the repository, ServiceRegistry class is used and this class is dependent on Service and CloudServer class. ServiceInvokation class is the main class to invoke 
software services hosted on the cloud server, from client web application. Based on the selection of cloud server and software services from the available lists, ServiceInvoke class will invoke Clustering/Classifier/Association software services. After invoking, service invocation results will be recorded and saved in the repository for the future usage.

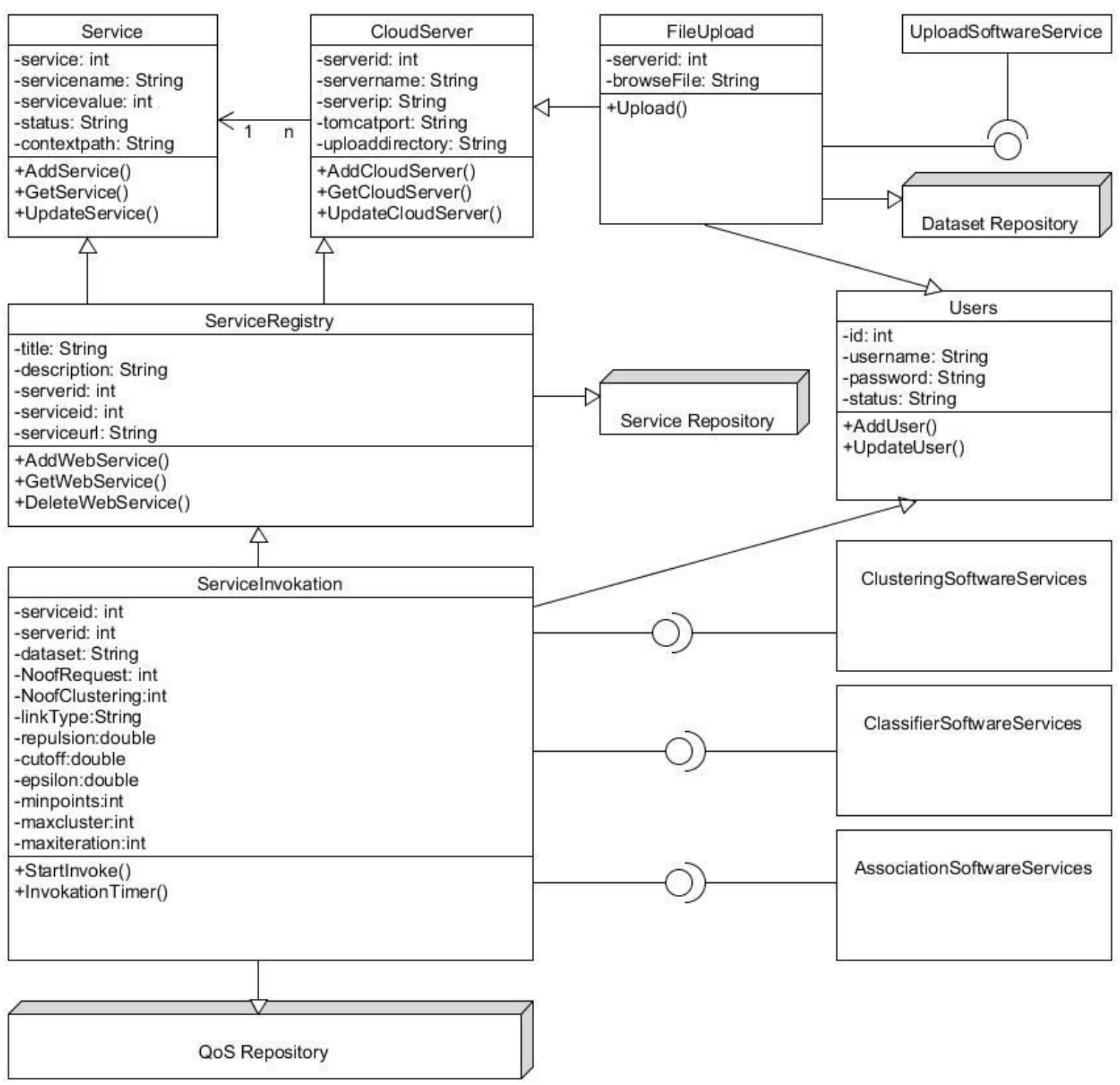

Figure 4.1: Class diagram of our implementation 


\subsubsection{User Interfaces}

In this section, we will show some of our major implemented user interfaces. These interfaces could illustrate the typical system workflows and user tasks. Figure 4.2 shows the interface for a user to upload datasets.

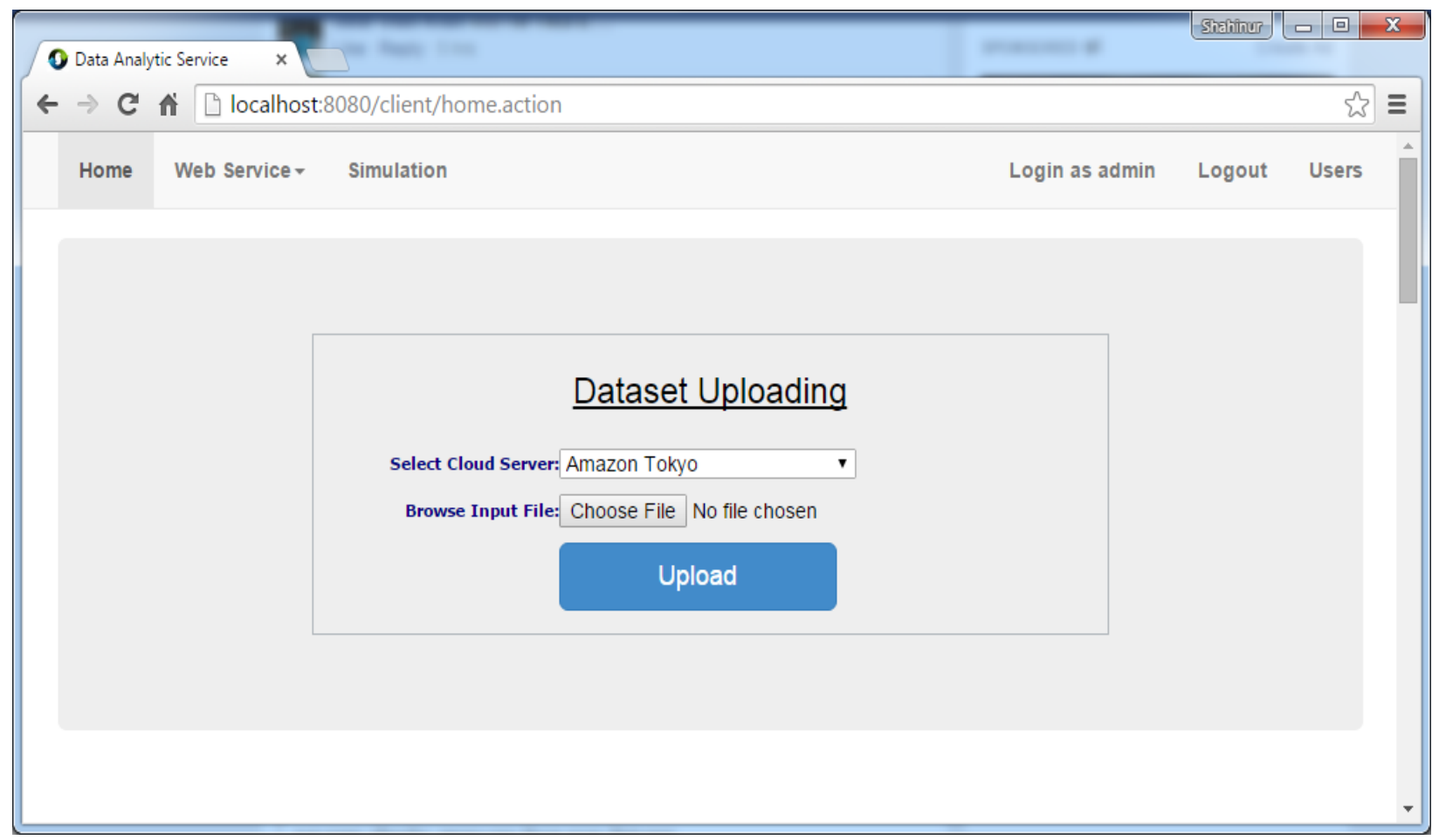

Figure 4.2: File upload UI

Figure 4.3 shows one of our major UIs called Cloud server setup. Using this interface, admin can set up a number of cloud servers in the system. According to the figure, cloud server name is simply the name of the server and this name must be unique. IP is the IP address of the cloud server, for example 127.0.0.1 and Tomcat port is the port of the installed Tomcat on the cloud server. The default Apache Tomcat port is 8080 , but it can be changed anytime. Upload Base Directory is a physical location in the cloud server where dataset will be stored, for example "C:/cloudupload/". Upload Directory is another location inside Upload Base Directory, 
for example amazon, dataset, etc. which is just a naming convention to make difference between one directory to another. R Installed Path is the location of the installation directory of R studio, for example "C:/program files/R/R-3.0.3/bin/Rscript.exe". This path is the most important path which is needed to invoke the R software services. This location is needed to execute the R script inside the Java code.

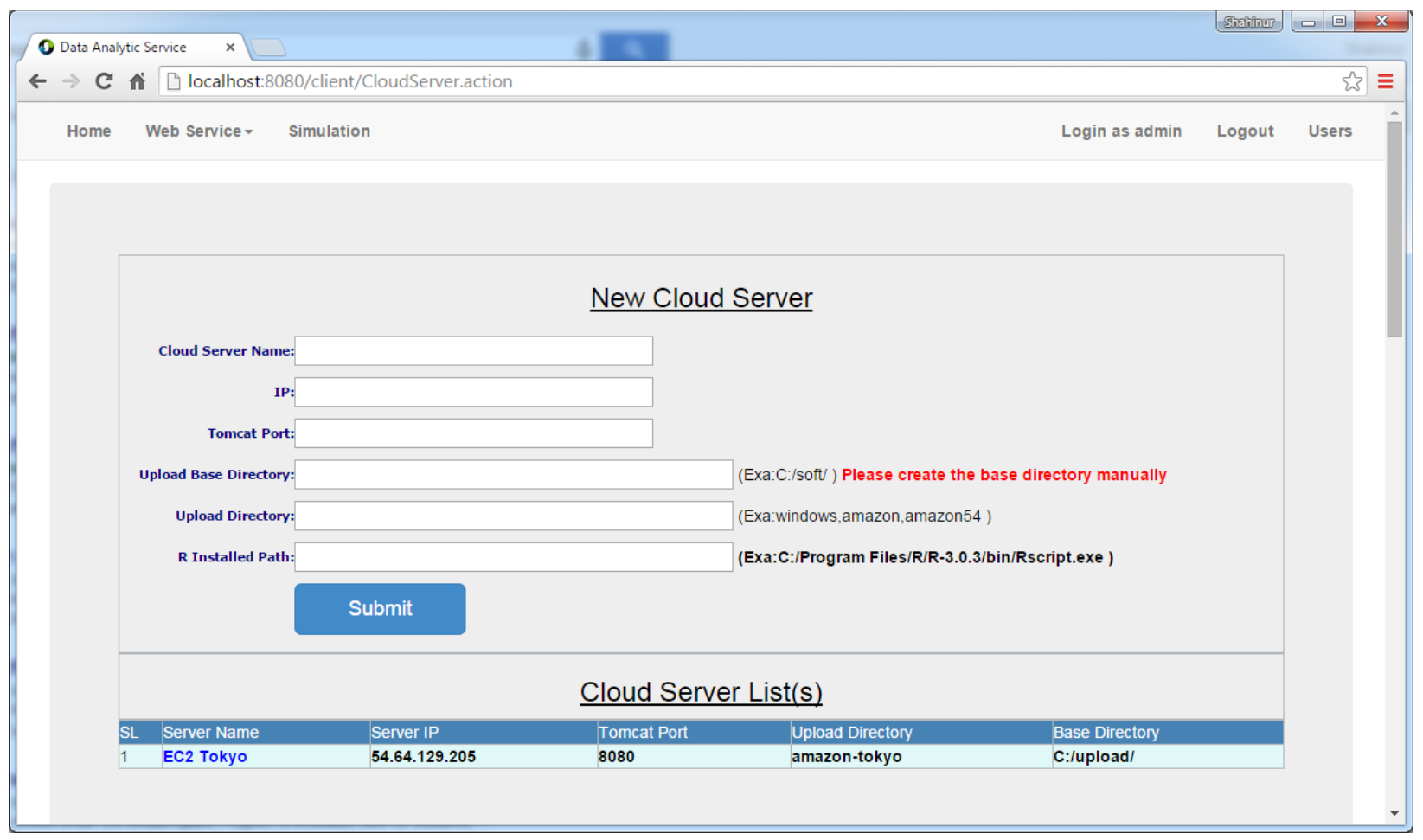

Figure 4.3: Cloud server setup UI

Figure 4.4 shows the interface for software service publication or registration into our system. Using this UI, admin or provider can publish their software services in the cloud. In the figure, title is the identifier or name of the service and it must be unique in the system. Description is not a mandatory field. Based on the selection of the cloud server and the service from the drop down list, system will generate invocation URL of the service, for example, if the context path of the service is "/emclustering/rest/emclustering" and IP of the cloud server is 
52.10.109.251 and apache tomcat server port is 8080, the generated URL for the service would be "http:// 52.10.109.251:8080/emclustering/rest/emclustering".

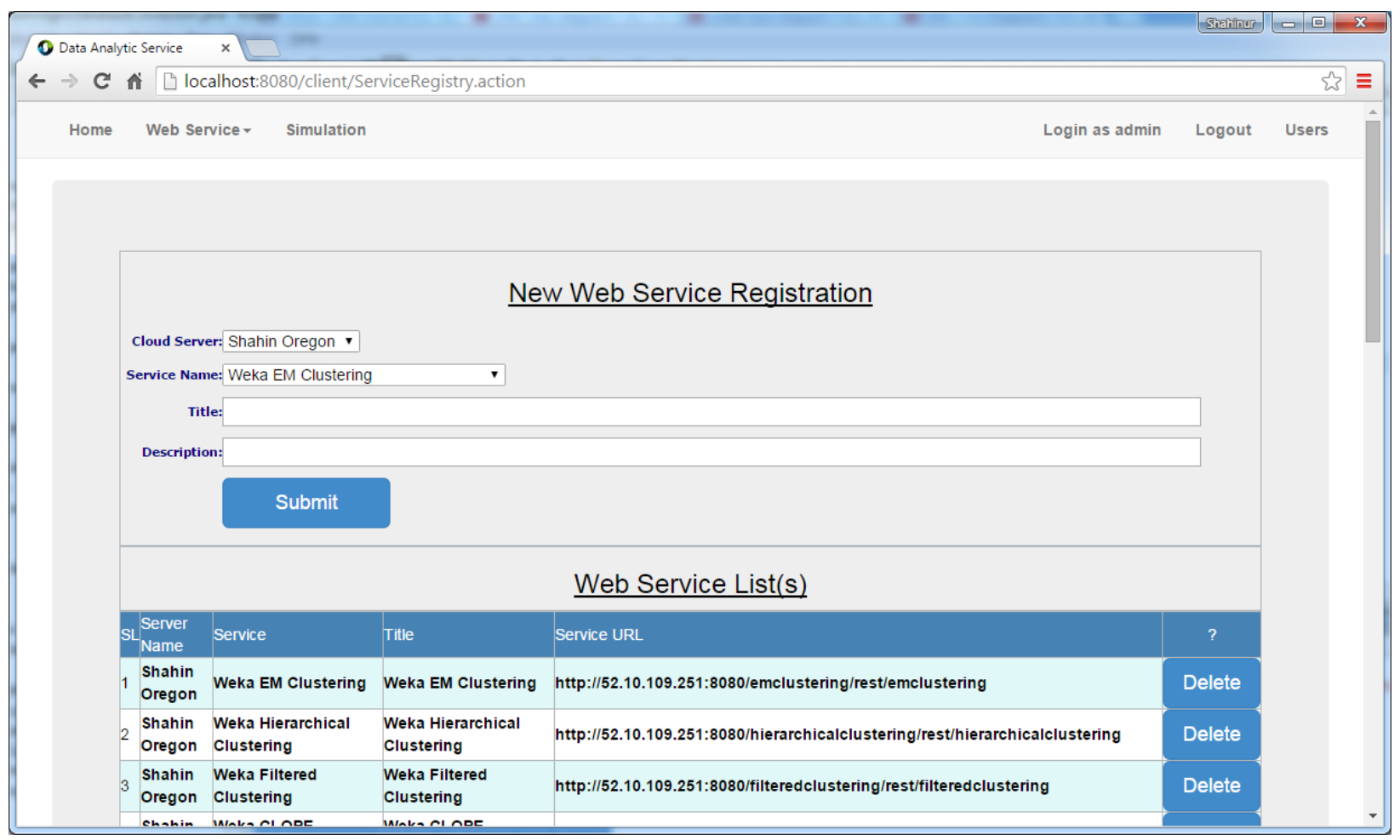

Figure 4.4: Web service publish or registration UI

Figure 4.5 shows the service invocation user interface. All the uploaded datasets, all the cloud servers and available published services are loaded into the drop down list for user to select a service to invoke. 


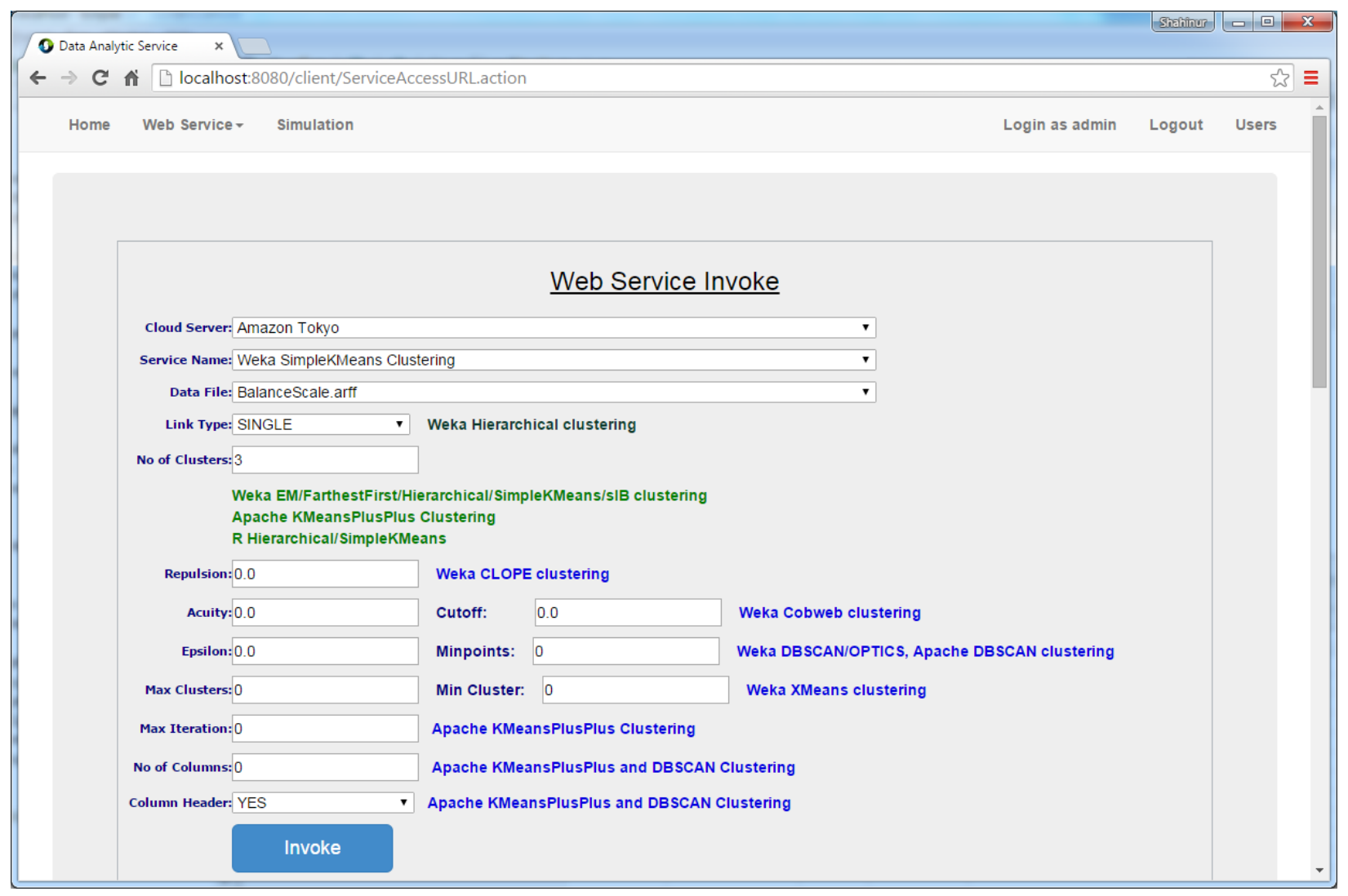

Figure 4.5: Web service invocation

In the above figure, there are a couple of parameters that users need to set their values. No of clusters means how many clusters user wants to form on his/her dataset. Repulsion parameter is used only for Weka CLOPE clustering algorithm and the default value is 2.6. The dataset for Weka CLOPE clustering should be nominal type, otherwise service invocation could fail. Users also need to set the repulsion parameter value very carefully because the parameter value affects directly the success of the service invocation. Acuity and Cutoff are the two parameters used for Weka Cobweb clustering. The default value of these two parameters are 1.0 and 0.0028. The success and failure of Weka Cobweb clustering service depend on the parameter values. Weka Cobweb clustering service supports both nominal and non-nominal values. Epsilon and Minpoints are two double type parameters used for Weka DBSCAN/Optics clustering and 
Apache DBSCAN clustering algorithms. The default values of these two parameters are 0.9 and 6 respectively. MaxCluster and MinCluster are Weka XMeans clustering parameters. The default values of MaxCluster and MinCluster are 4 and 2 respectively. MaxIteration parameter is used for Apache KMeansPlusPlus clustering which means how many times the system will iterate on the dataset to get the expected output. For invocation of apache clustering service, users can decide whether to use the header information on the dataset. If they use header information, they need to set YES of Column Header parameter. If Column header is set to YES, service will ignore that line to apply the clustering algorithm on the dataset. All these parameters have default values. If users are not familiar with the algorithm, they can always use the default values. If they have some knowledge about the algorithm, they can set their own values good for their datasets.

Table 4.2 shows a list of parameters that users can pass before invoking services and the corresponding services which require them.

Table 4.2: Software service parameters

\begin{tabular}{|ll|}
\hline Parameter Name & Applicable Services \\
\hline No of cluster & Weka EM/FarthestFirst/Hierarchical/SimpleKMeans/sIB Clustering, Apache \\
& KMeansPlusPlus Clustering, R Hierarchical/ SimpleKMeans Clustering \\
\hline Repulsion & Weka CLOPE Clustering \\
\hline Acuity, Cutoff & Weka Cobweb Clustering \\
\hline Epsilon, Minpoints & Weka DBSCAN/OPTICS Clustering, Apache DBSCAN Clustering \\
\hline Max(Min)Cluster & Weka XMeans Clustering \\
\hline Max Iteration & Apache KMeansPlusPlus Clustering \\
\hline No of columns & Apache KMeansPlusPlus/DBSCAN Clustering \\
\hline Column Header & Apache KMeansPlusPlus/DBSCAN Clustering \\
\hline
\end{tabular}


Figure 4.6 shows service invocation result using Weka SimpleKMeans clustering service. It also shows the percentage of incorrectly clustered instances and the latency value in milliseconds (ms).

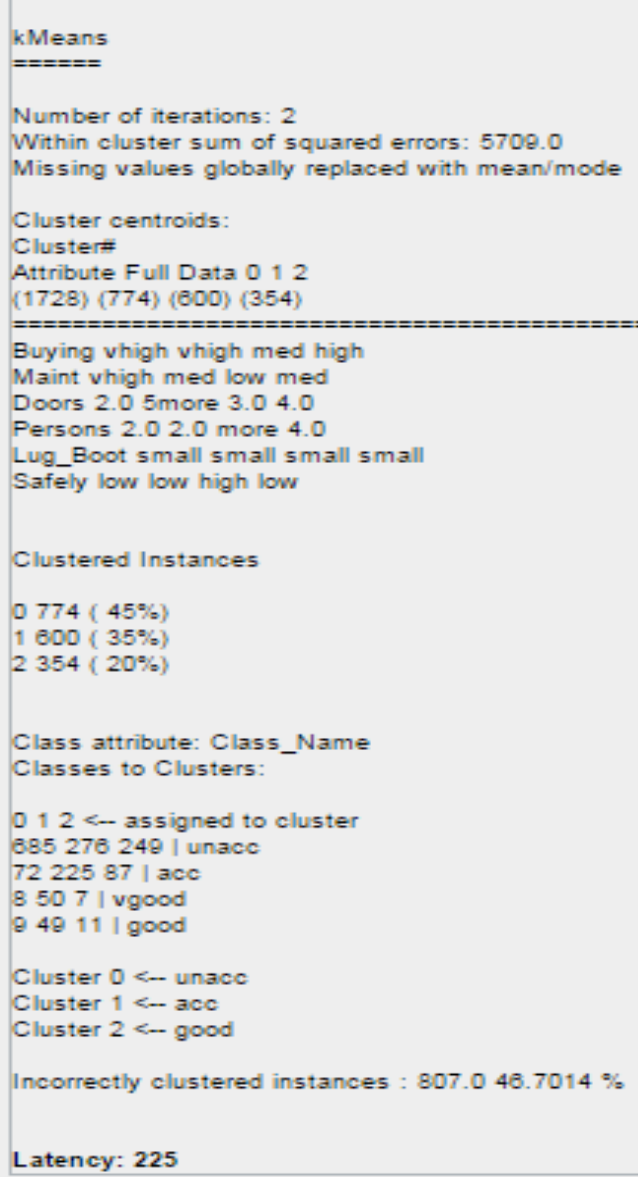

Figure 4.6: Service invocation result

Figure 4.7 shows the end-to-end QoS values for Weka SimpleKMeans clustering service based on the dataset used.

\begin{tabular}{|c|c|c|c|c|c|c|c|}
\hline \multicolumn{8}{|c|}{ Web Service Invoke Result } \\
\hline ip of Request & If of Success & If of Failure & Response Time & Availability(\%) & Throughput & Reliability(\%) & Latency \\
\hline 1 & 1 & 0 & 3.25 & 100 & 3.594 & 100 & 0.225 \\
\hline
\end{tabular}

Figure 4.7: Collected QoS values 
Latency is measured as the time taken for the cloud server to process a given request and it is 0.225 seconds. Response time is calculated as the ping time to the server plus the latency. Reliability is the percentage of the success request. For this one request, the invocation is success and Reliability is $100 \%$. As the system and the service are running properly and invocation has been done successfully, Availability is also $100 \%$. Throughput has been calculated based on the output size of the service. The output size in byte divided by 1024, gives the output size in kilobyte. This output size in kilobyte is multiplied by 8 , which gives the output in kilobit. Then it is divided by total time taken for the successful request, which gives the Throughput value. The calculation steps are shown below,

Output size in byte $=903$

Output size in Kilobyte $=903 / 1024$

$=0.8818359375$

Output size in kilobit $=0.8818359375 * 8$

$$
=7.0546875
$$

Total time for successful request in seconds $=$ Response Time + Latency

$$
=3.188+0.168=3.356
$$

Throughput $=7.0546875 / 3.356=2.10211189$

\subsection{Proof of Concept Prototype}

The implemented platform is supposed to be generic, and can be used to include any number and type of services. In this work, we implemented a proof of concept prototype to showcase the value of the testbed platform and demonstrate how it is used to host data mining services in particular. 


\subsubsection{Data Mining Packages}

Data mining is the procedure of analyzing large quantities of data from different aspects and summarizing that data into useful format which is used further for the business purposes. Data mining techniques are used by different types of companies from medium sized to large sized companies to analyze their data and to take suitable decisions for their business growth. Data mining package or software is one kind of tool for analyzing the data. This tool allows users to analyze their data from different dimensions, categorize it and finally summarize it in a human readable format. Among many data mining algorithms, three categories of algorithms are most popular including Clustering, Classification and Association rule mining. Classifiers are used to allocate data to predetermined groups. Clustering is used to group data according to customer preferences or logical relationships. Association is used to mine data to identify associations between different items. Among various packages, we chose three for our work which are Weka, $\mathrm{R}$ and Apache Commons Math Machine Learning. The idea is that different packages represent different software providers, and in this way, we could have data mining services implementing the same algorithm coming from different providers.

Weka (Waikato Environment for Knowledge Analysis) is a popular data mining package, which is written in Java. Weka is a free software package under GNU General Public License. Weka contains a collection of visualization tools and algorithms for data analysis including graphical user interfaces to access the functionalities. Weka provides several standard data mining algorithms such as Preprocessing, Classification, Clustering, Association rule mining, Regression and Feature selection. Weka has the support of database access using Java Database Connectivity. This feature supports to process data returned by a database query. The main user interface of Weka is called Explorer. Users can use Explorer to run different data mining 
algorithms. In this work, instead of using this interface, we will use the Weka API to implement the services.

Table 4.3 shows a list of Weka algorithms that we chose to implement our services.

Table 4.3: List of Implemented Weka Algorithms

\begin{tabular}{|lll|}
\hline SL & Algorithm Name & Type \\
\hline $\mathbf{1}$ & Expectation Maximization (EM) & Clustering \\
\hline $\mathbf{3}$ & Hierarchical & Clustering \\
\hline $\mathbf{4}$ & Filtered & Clustering \\
\hline $\mathbf{5}$ & FarthestFirst & Clustering \\
\hline $\mathbf{6}$ & CLOPE & Clustering \\
\hline $\mathbf{7}$ & SimpleKMeans & Clustering \\
\hline $\mathbf{8}$ & Cobweb & Clustering \\
\hline $\mathbf{9}$ & sIB & Clustering \\
\hline $\mathbf{1 0}$ & DBSCAN & Clustering \\
\hline $\mathbf{1 1}$ & XMeans & Clustering \\
\hline $\mathbf{1 2}$ & BFTree & Classifier \\
\hline $\mathbf{1 3}$ & AdaBoostM1 & Classifier \\
\hline $\mathbf{1 4}$ & LMT & Classifier \\
\hline $\mathbf{1 5}$ & REPTree & Classifier \\
\hline $\mathbf{1 6}$ & RandomTree & Classifier \\
\hline $\mathbf{1 7}$ & RandomForest & Classifier \\
\hline $\mathbf{1 8}$ & Association \\
\hline
\end{tabular}

$\mathrm{R}[41]$ is an open source programming language and software environment for statistical calculation and data analysis. $\mathrm{R}$ has many interfaces to communicate with the code which is 
written in other programming language such as $\mathrm{C}, \mathrm{C}++$, Fortran, Java and Python. $\mathrm{S}$ is a statistical programming language and $\mathrm{R}$ is an implementation of $\mathrm{S}$ programming language. The $\mathrm{R}$ language is widely used by statisticians and data miners for statistical software development. R implements a wide range of statistical and graphical techniques including clustering, classification for statistics data analysis. As we used Java programming language for developing the web services and $\mathrm{R}$ is a different programming language, we need an intermediate interpreter that will communicate with $\mathrm{R}$ compiler and execute the $\mathrm{R}$ script within Java. For this purpose we chose RCaller as an intermediate interpreter.

Table 4.4 shows the list of our implemented $\mathrm{R}$ algorithms. We mainly focus on clustering algorithms here from $\mathrm{R}$ package.

Table 4.4: List of Implemented R algorithms

\begin{tabular}{|lll|}
\hline SL & Algorithm Name & Type \\
\hline $\mathbf{1}$ & Hierarchical & Clustering \\
\hline $\mathbf{2}$ & KMeans & Clustering \\
\hline
\end{tabular}

Apache Commons Math Machine Learning is a project of Apache Software Foundation and its purpose is to provide reusable open source software. Apache Commons Mathematics has implemented some machine learning algorithms which are open source and written in Java. Apache Software Foundation has implemented four clustering algorithms which are KMean++, Fuzzy-KMeans, DBSCAN and Multi-KMeans++.

Table 4.5 shows the list of Apache Commons Mathematics machine learning algorithms used to develop services. Here we only consider clustering algorithm. 
Table 4.5: List of implemented Apache Commons Mathematics machine learning algorithms

\begin{tabular}{|lll|}
\hline SL & Algorithm Name & Type \\
$\mathbf{1}$ & KMeans++ & Clustering \\
\hline $\mathbf{2}$ & DBSCAN & Clustering \\
\hline
\end{tabular}

So in this prototype implementation, we have 3 software providers - Weka, R, and Apache Commons Math Machine Learning. There are 3 types of data mining services clustering, classification, and association rule mining. Weka services cover all three types, Apache services only cover clustering services and $\mathrm{R}$ services also only cover clustering services. Although a few clustering algorithms have been implemented in both $\mathrm{R}$ and Apache, here we only choose two from each which we can find corresponding implementations in Weka. In this way, we could test the impact of different factors (provider, functionality, algorithms) of SaaS services on the QoS values.

\subsubsection{IaaS Services}

Since we need to have multiple IaaS services from multiple providers, in this work we chose Amazon EC2 and Windows Azure cloud infrastructure to host our developed software services. Then for services from each provider, we also want to have different configurations and different hosting locations, so that we could have a variety of IaaS services. Amazon EC2, which is also known as Amazon Elastic Compute Cloud, provides resizable computing capacity in the Amazon Web Services (AWS) cloud. Amazon EC2 offers a wide range of instance types that vary based on combinations of CPU power, amount of memory, storage size and network capacity. Amazon provides pre-configured instances, which is known as Amazon Machine Images (AMIs) that include operating systems such as Windows or Linux. The AMIs may 
include a wide range of preinstalled software packages. Amazon EC2 provides various types of instances based on the workload from "micro" instances for small jobs to high performance "xlarge" instances for data warehousing tasks. The users can also mix different types of instances based on the amount of memory they need. Amazon EC2 Spot Instances allow users to bid on spare instances to be used whenever user's bid price exceeds the current spot price. Amazon EC2 Reserved Instances provide customers a good discount which could be up to $75 \%$. Amazon EC2 Dedicated Instances is another instance type which is based on pay-as-you-go pricing model and there is no long-term commitment.

For our work, we chose the Amazon EC2 Dedicated Instances to host our developed web services in Windows platform. There are a number of options in this type and we chose the micro type for our thesis.

Table 4.6 shows our chosen Amazon EC2 IaaS configuration to host our developed software services.

Table 4.6: Amazon EC2 IaaS configuration

\begin{tabular}{|llllll|}
\hline SL & Name & OS \& Bit & Processor & RAM & Location \\
\hline $\mathbf{1}$ & Amazon-1 & Windows Server 2012 R2 (64 bit) & Intel(R) @ 2.5 GHz & 1 GB & Tokyo \\
\hline $\mathbf{2}$ & Amazon-2 & Windows Server 2012 R2 (64 bit) & Intel(R) @ 2.5 GHz & 1 GB & US (Oregon) \\
\hline $\mathbf{3}$ & Amazon-3 & Windows Server 2003 R2 (32 bit) & Intel (R) @ 2.5 GHz & 4 GB & US (Oregon) \\
\hline
\end{tabular}

Windows Azure is a Microsoft implemented cloud computing platform. Windows Azure was renamed to Microsoft Azure on April, 2014 [45]. Windows Azure uses Fabric layer, which is a cluster hosted in Microsoft data center that manages computing resources. The two important features of Windows Azure are scaling and reliability which are controlled by Microsoft Azure 
Fabric Controller in such a way that services and environments do not crash if any of the services goes down. A feature called Automated Service Management supports application upgrading without compromising performance. Windows Azure provides a number of services such as data management, media services, networking, virtual network, virtual machine, integration among applications, high density website hosting, and access control service, etc. Windows Azure supports a variety of Microsoft and third-party protocols, programming languages and platforms including XML, REST, SOAP, Eclipse, Ruby, PHP, Python, etc.

As Amazon-1 and Amazon-2 instances are under free tier account, we cannot chose more RAM for the instances. But as Amazon-3 is a paid instance, we selected 4 GB RAM. We could not select same type of IaaS from Amazon and Azure because of their different infrastructure configuration. For example Windows Azure has core options but Amazon does not have that. As a free user, we can select maximum 1 GB RAM from Amazon but 4 GB RAM from Windows Azure.

Table 4.7 shows our chosen Windows Azure IaaS configuration for hosting our developed software services. All Azure instances are under one month free subscription and we have to select at least 4 cores. This is how Windows Azure are configured.

Table 4.7: Windows Azure IaaS configuration

\begin{tabular}{|llllll|}
\hline SL & Name & OS \& Bit & Processor & RAM & Location \\
\hline $\mathbf{1}$ & Azure-1 & Windows Server 2008 R2 (64 bit) & 7 core & 14GB & Japan West \\
\hline $\mathbf{2}$ & Azure-2 & Windows Server 2008 R2 (64 bit) & 7 core & 14GB & North Europe \\
\hline $\mathbf{3}$ & Azure-3 & Windows Server 2008 R2 (64 bit) & 4 core & 7GB & Central US \\
\hline $\mathbf{4}$ & Azure-4 & Windows Server 2012 R2 (64 bit) & 4 core & 14GB & Central US \\
\hline
\end{tabular}


As we have mainly used free tier of the IaaS account and different cloud service providers have different types of options, we are limited in our IaaS configuration selection. For example as a free tier user, we cannot select more memory in Amazon EC2. Amazon does not provide core configuration to the free user. On the other hand, Windows Azure gives core options available to their users.

\subsection{QoS Data Collection}

In this work, we use the real datasets collected from UCI machine learning repository [46]. Based on the task type, there are a number of different types of datasets. Each dataset may have different data properties, for example, different sizes, different dimensions, different types of data (numeric, categorical, Boolean, etc.). Here we chose six of them for our experiment because they are varying on these data properties.

Table 4.8 describes our used datasets in this work. We chose our datasets based on four criteria: the number of dimensions, size (number of rows), whether to have missing data or not, and the data type. The target was to find datasets with varying values on these criteria. The required information was obtained from the UCI dataset description.

Table 4.8: List of datasets

\begin{tabular}{|llrrrrr|}
\hline SL & Dataset Name & \# of rows & Dimension & Missing Values & Year & Data Type \\
\hline $\mathbf{1}$ & Lung Cancer & 32 & 56 & Yes & 1992 & numeric, string \\
\hline $\mathbf{2}$ & Balance Scale & 625 & 4 & No & 1994 & nominal \\
\hline $\mathbf{3}$ & Car Evaluation & 1728 & 6 & No & 1997 & numeric, string \\
\hline $\mathbf{4}$ & Turkiye student evaluation & 5820 & 33 & No & 2013 & numeric \\
\hline $\mathbf{5}$ & Opt digits & 5620 & 64 & No & 1998 & numeric \\
\hline $\mathbf{6}$ & Diabetic data & 100000 & 55 & Yes & 2014 & numeric \\
\hline
\end{tabular}


We developed a client web application for our experiment. Its functions include setting up the cloud server, publishing software services, uploading user's datasets, invoking software services and monitoring software services' QoS values. For experiment, we hosted our developed software services into different cloud infrastructure services from either Windows Azure or Amazon EC2. Then we hosted client web application in our local system. We used default parameter values for classifier and association rule mining services. We used some default parameter values for clustering algorithm and we also implemented some parameters into the client web application where users can send his/her chosen parameter values before invoking software services.

In our experiment, we set a threshold value for time-out. If the client web application does not receive any response from a service within five minutes, that invocation will be timed out. We sent ten requests to invoke a services for all IaaS and datasets from the client application. Users can send more than ten requests. First of all, an end-user needs to upload his/her own dataset to the desired cloud server. To invoke a service, the end-user needs to select cloud server, software service and dataset from the drop down list. Then user also needs to tell the system how many requests he/she wants to send to the server to invoke the service. After that, user can enter available parameter values or use default values. The parameter values could affect whether we could get proper result from that service. If wrong parameter values are provided, some QoS values can be affected or sometimes service invocation could fail.

We show some of the collected QoS values below. In these tables, status 0 means a successful invocation and status -1 means a failed invocation. When status is -1 , the other 
columns have empty cells. For all the software services, we have availability $100 \%$ and therefore this value is not included in the tables.

Table 4.9 shows QoS values of all implemented software services hosted on the same IaaS service (Azure-1) running for the same dataset (i.e. Balance scale dataset). From this table, we can see that among all clustering services, Weka Hierarchical Clustering, Weka DBSCAN Clustering, Apache KMeansPlusPlus Clustering, Apache DBSCAN Clustering, R Hierarchical Clustering and R KMeans Clustering failed to process the dataset. The reason of failure for Weka DBSCAN clustering is selection of the wrong parameter values for Epsilon and Minpoints. If these two values are not provided properly, algorithm will fail to process the file. As Balance scale dataset contains string data, the other listed services failed to process the file because they only support numeric values. Weka Apriori Associator and Weka Tertius Associator failed because they only support nominal data type. We could also see that although the services are applied on the same dataset and hosted on same IaaS instance, the collected QoS values vary between different SaaS services.

Table 4.10 shows QoS values of all implemented software services hosted on the same IaaS service (Azure-1) running for another dataset (i.e. Lung Cancer). 
Table 4.9: QoS values for one dataset (Balance Scale), one IaaS (Azure-1) and all services

\begin{tabular}{|c|c|c|c|c|c|}
\hline Service Name & Status & Response Time (s) & Throughput & Reliability & Latency(s) \\
\hline Weka EM Clustering & 0 & 4.133 & 1.451 & $100 \%$ & 0.272 \\
\hline Weka Hierarchical Clustering & -1 & & & & \\
\hline Weka Filtered Clustering & 0 & 3.644 & 2.534 & $100 \%$ & 0.019 \\
\hline Weka CLOPE Clustering & 0 & 4.111 & 4.177 & $100 \%$ & 0.136 \\
\hline Weka FarthestFirst Clustering & 0 & 3.791 & 0.857 & $100 \%$ & 0.036 \\
\hline Weka SimpleKMeans Clustering & 0 & 3.845 & 1.805 & $100 \%$ & 0.061 \\
\hline Weka Cobweb Clustering & 0 & 4.111 & 4.017 & $100 \%$ & 0.136 \\
\hline Weka sIB Clustering & 0 & 4.449 & 1.864 & $100 \%$ & 0.537 \\
\hline Weka XMeans Clustering & 0 & 4.244 & 1.460 & $100 \%$ & 0.041 \\
\hline Weka DBSCAN Clustering & -1 & & & & \\
\hline Apache KMeansPlusPlus Clustering & -1 & & & & \\
\hline Apache DBSCAN Clustering & -1 & & & & \\
\hline R Hierarchical Clustering & -1 & & & & \\
\hline R KMeans Clustering & -1 & & & & \\
\hline Weka BFTree Classifier & 0 & 3.999 & 1.844 & $100 \%$ & 0.097 \\
\hline Weka AdaBoostM1 Classifier & 0 & 3.917 & 1.910 & $100 \%$ & 0.038 \\
\hline Weka REPTree Classifier & 0 & 3.962 & 1.895 & $100 \%$ & 0.025 \\
\hline Weka LMT Classifier & 0 & 5.385 & 1.085 & $100 \%$ & 1.575 \\
\hline Weka RandomTree Classifier & 0 & 3.810 & 1.975 & $100 \%$ & 0.016 \\
\hline Weka RandomForest Classifier & 0 & 4.095 & 1.828 & $100 \%$ & 0.038 \\
\hline Weka Apriori Associator & -1 & & & & \\
\hline Weka Tertius Associator & -1 & & & & \\
\hline
\end{tabular}


Table 4.10: QoS values for one dataset (Lung Cancer), one IaaS (Azure-1) and all services

\begin{tabular}{|c|c|c|c|c|c|}
\hline Service Name & Status & Response Time (s) & Throughput & Reliability & Latency(s) \\
\hline Weka EM Clustering & 0 & 3.907 & 9.168 & $100 \%$ & 0.042 \\
\hline Weka Hierarchical Clustering & 0 & 3.866 & 1.902 & $100 \%$ & 0.003 \\
\hline Weka Filtered Clustering & 0 & 3.869 & 9.095 & $100 \%$ & 0.008 \\
\hline Weka CLOPE Clustering & 0 & 4.869 & 0.839 & $100 \%$ & 0.295 \\
\hline Weka FarthestFirst Clustering & 0 & 3.873 & 2.077 & $100 \%$ & 0.003 \\
\hline Weka SimpleKMeans Clustering & 0 & 3.542 & 8.150 & $100 \%$ & 0.005 \\
\hline Weka Cobweb Clustering & 0 & 4.132 & 0.706 & $100 \%$ & 0.051 \\
\hline Weka sIB Clustering & -1 & & & & \\
\hline Weka XMeans Clustering & 0 & 3.918 & 4.675 & $100 \%$ & 0.109 \\
\hline Weka DBSCAN Clustering & -1 & & & & \\
\hline $\begin{array}{l}\text { Apache KMeansPlusPlus } \\
\text { Clustering }\end{array}$ & 0 & 3.803 & 4.210 & $100 \%$ & 0.006 \\
\hline Apache DBSCAN Clustering & 0 & 3.923 & 0.075 & $100 \%$ & 0.02 \\
\hline R Hierarchical Clustering & 0 & 4.102 & 12.533 & $100 \%$ & 0.328 \\
\hline R KMeans Clustering & 0 & 3.909 & 9.345 & $100 \%$ & 0.19 \\
\hline Weka BFTree Classifier & 0 & 3.617 & 1.825 & $100 \%$ & 0.003 \\
\hline Weka AdaBoostM1 Classifier & 0 & 3.942 & 1.673 & $100 \%$ & 0.008 \\
\hline Weka REPTree Classifier & 0 & 3.984 & 1.658 & $100 \%$ & 0 \\
\hline Weka LMT Classifier & 0 & 4.063 & 1.605 & $100 \%$ & 0.055 \\
\hline Weka RandomTree Classifier & 0 & 3.819 & 1.730 & $100 \%$ & 0 \\
\hline Weka RandomForest Classifier & 0 & 3.596 & 1.836 & $100 \%$ & 0.003 \\
\hline Weka Apriori Associator & -1 & & & & \\
\hline Weka Tertius Associator & -1 & & & & \\
\hline
\end{tabular}


From this table, we can see that among all clustering services, Weka sIB Clustering, Weka DBSCAN Clustering, Weka Apriori Association and Weka Tertius Association failed to process the dataset. The reason of failure for Weka DBSCAN clustering is selection of the wrong parameter values for Epsilon and Minpoints. Weka Apriori Associator and Weka Tertius Associator failed because Lung Cancer dataset contains numeric data but these two associators only support nominal data type. Comparing Table 4.9 and Table 4.10, we can see we have higher success rate in Table 4.10, which means successful invocation depends on dataset, data types and parameter values also.

Table 4.11 shows the average QoS values on one IaaS (Azure-1) for all datasets. The QoS values are averaged on all SaaS services.

Table 4.11: Average QoS values on one IaaS (Azure-1) for all datasets

\begin{tabular}{|lcccr|}
\hline Dataset Name & Latency (Sec) & Reliability & Response Time(Sec) & Throughput \\
\hline Lung Cancer & 0.054 & $81.81 \%$ & 3.929 & 4.061 \\
\hline Balance Scale & 0.168 & $63.64 \%$ & 4.106 & 1.886 \\
\hline Car Evaluation & 1.952 & $54.54 \%$ & 6.819 & 1.771 \\
\hline Opt digits & 10.318 & $68.18 \%$ & 11.882 & 4.236 \\
\hline Turkiye student evaluation & 6.332 & $68.18 \%$ & 8.819 & 2.837 \\
\hline Diabetic data & 24.354 & $31.81 \%$ & 13.329 & 0.180 \\
\hline
\end{tabular}

From the above table, we can observe the average reliability of the services on Diabetic dataset is less than that on other datasets because diabetic dataset has a lot of missing data and noise. We can also see that the reliability on Lung cancer dataset is more than that on other dataset because this dataset is small and clean. We could see that Lung Cancer dataset has the 
smallest latency because it is the smallest based on its size and dimension, and Diabetic dataset has the largest latency because it is the biggest dataset among all. The response time is also affected by the dataset size and dimension. But it is affected more heavily by the network transmission time, which explains why the response time is much bigger than the latency. There is no clear pattern on throughput because throughput is not only decided by the service processing time, the network transmission time, but also decided by the size of the output which varies a lot among different software services. We observe the similar patterns for other IaaS services, and thus we are not showing their values here.

Table 4.12 shows the average QoS values of one SaaS (Weka FarthestFirst Clustering) for all datasets. The QoS values are averaged on all IaaS services.

Table 4.12: Average QoS values of one SaaS (Weka FarthestFirst Clustering) for all datasets

\begin{tabular}{|lcccr|}
\hline Dataset Name & Latency (Sec) & Reliability & Response Time(Sec) & Throughput \\
\hline Lung Cancer & 0.053 & $100 \%$ & 3.625 & 2.218 \\
\hline Balance Scale & 0.054 & $100 \%$ & 3.612 & 0.903 \\
\hline Car Evaluation & 0.089 & $100 \%$ & 3.736 & 1.033 \\
\hline Opt digits & 3.079 & $100 \%$ & 9.466 & 1.818 \\
\hline Turkiye student evaluation & 6.830 & $100 \%$ & 16.674 & 1.081 \\
\hline Diabetic data & 2.093 & $100 \%$ & 5.638 & 1.164 \\
\hline
\end{tabular}

From the above table, we can see the Reliability of Weka FarthestFirst Clustering service for all datasets is $100 \%$. Interestingly, this service works very fast for Diabetic dataset. Although it is the biggest dataset, its latency is lower than two other smaller datasets. The latency for Turkiye student evaluation dataset is slower than those for other datasets. 
Table 4.13 shows the average QoS values on all IaaS for one dataset (Lung Cancer). The QoS values are averaged on all SaaS services.

Table 4.13: Average QoS values for one dataset (Lung Cancer) on all IaaS

\begin{tabular}{|lcccr|}
\hline IaaS Name & Latency(sec) & Reliability & Response Time(sec) & Throughput \\
\hline Azure-1 & 0.054 & $81.81 \%$ & 4.115 & 4.217 \\
\hline Azure-2 & 0.066 & $81.81 \%$ & 3.489 & 2.858 \\
\hline Azure-3 & 0.848 & $81.81 \%$ & 4.478 & 4.017 \\
\hline Azure-4 & 0.785 & $81.81 \%$ & 4.521 & 3.654 \\
\hline Amazon-1 & 1.247 & $81.81 \%$ & 4.283 & 2.570 \\
\hline Amazon-2 & 1.434 & $81.81 \%$ & 4.771 & 2.852 \\
\hline Amazon-3 & 1.282 & $81.81 \%$ & 4.924 & 3.043 \\
\hline
\end{tabular}

In this table, we can see the reliability is the same $(81.81 \%)$ for all the IaaS services, which means the reliability is mainly decided by the dataset features. We can also see the variation of latency and throughput, which shows the impact of IaaS configurations and locations on their values. Since Azure instances have higher configurations, in general, their latency values are better than those from EC2 instances. The impact of configurations on response time is smaller because the network transmission time is usually bigger than the processing time, and it is mainly decided by the location. So the instances from Europe or Asia usually have longer response time. Throughput also varied among different IaaS instances, however no clear pattern can be observed.

Table 4.14 shows the average QoS of one SaaS (Weka SimpleKMeans Clustering) on all IaaS. The QoS values are averaged on all the datasets. 
Table 4.14: Average QoS values of one SaaS (Weka SimpleKMeans Clustering) on all IaaS

\begin{tabular}{|lcccr|}
\hline IaaS Name & Latency(sec) & Reliability & Response Time(sec) & Throughput \\
\hline Azure-1 & 13.165 & $100 \%$ & 17.067 & 3.680 \\
\hline Azure-2 & 10.145 & $100 \%$ & 13.563 & 4.339 \\
\hline Azure-3 & 19.023 & $100 \%$ & 22.105 & 3.696 \\
\hline Azure-4 & 19.107 & $100 \%$ & 21.818 & 3.996 \\
\hline Amazon-1 & 0.199 & $83.33 \%$ & 3.359 & 5.335 \\
\hline Amazon-2 & 0.308 & $83.33 \%$ & 3.745 & 4.724 \\
\hline Amazon-3 & 0.247 & $83.33 \%$ & 3.359 & 5.294 \\
\hline
\end{tabular}

From this table, we observed that the Latency and Response time of Amazon IaaS are lower than that of Azure IaaS. We have one big size dataset called Diabetic which was invoked successfully in Azure IaaS but failed in Amazon IaaS due to time-out. Invocation for Azure succeeded because of its good configuration but it took long time to finish the invocation. On the other hand, Amazon IaaS timed out to process Diabetic dataset due to its low configuration. The way we calculated the latency is to remove the failed cases. So with exclusion of this dataset, Amazon IaaS gives better latency values. However, this failure also affects the reliability. The Amazon IaaS has lower reliability than Azure IaaS.

Table 4.15 shows the average QoS values of all SaaS for one dataset (Lung Cancer). The QoS values are averaged on all IaaS instances. For the fail cases, their columns have empty values. 
Table 4.15: Average QoS values of all SaaS for one dataset (Lung Cancer)

\begin{tabular}{|c|c|c|c|c|}
\hline Service Name & Latency (sec) & Response Time (sec) & Reliability & Throughput \\
\hline Weka EM Clustering & 0.062 & 3.462 & $100 \%$ & 10.384 \\
\hline \multicolumn{5}{|l|}{ Weka Hierarchical Clustering } \\
\hline Weka Filtered Clustering & 0.043 & 3.608 & $100 \%$ & 9.809 \\
\hline Weka CLOPE Clustering & 0.295 & 4.869 & $100 \%$ & 0.387 \\
\hline Weka FarthestFirst Clustering & 0.002 & 3.509 & $100 \%$ & 2.055 \\
\hline Weka SimpleKMeans Clustering & 0.034 & 3.302 & $100 \%$ & 7.503 \\
\hline Weka Cobweb Clustering & 0.051 & 4.132 & $100 \%$ & 0.743 \\
\hline \multicolumn{5}{|l|}{ Weka sIB Clustering } \\
\hline Weka XMeans Clustering & 0.097 & 3.809 & $100 \%$ & 4.033 \\
\hline \multicolumn{5}{|l|}{ Weka DBSCAN Clustering } \\
\hline Apache KMeansPlusPlus Clustering & 0.104 & 3.667 & $42.85 \%$ & 4.995 \\
\hline Apache DBSCAN Clustering & 0.020 & 3.822 & $42.85 \%$ & 0.082 \\
\hline R Hierarchical Clustering & 0.422 & 4.133 & $42.85 \%$ & 11.903 \\
\hline R KMeans Clustering & 0.304 & 3.775 & $42.85 \%$ & 12.605 \\
\hline Weka BFTree Classifier & 0.004 & 3.553 & $100 \%$ & 1.605 \\
\hline Weka AdaBoostM1 Classifier & 0.116 & 3.503 & $100 \%$ & 1.673 \\
\hline Weka REPTree Classifier & 0.005 & 3.807 & $100 \%$ & 1.709 \\
\hline Weka LMT Classifier & 0.115 & 3.993 & $100 \%$ & 1.673 \\
\hline Weka RandomTree Classifier & 0.104 & 3.504 & $100 \%$ & 1.773 \\
\hline Weka RandomForest Classifier & 0.204 & 3.604 & $100 \%$ & 1.803 \\
\hline \multicolumn{5}{|l|}{ Weka Apriori Associator } \\
\hline Weka Tertius Associator & & & & \\
\hline
\end{tabular}


From this table, we can see that among all the clustering services, Weka FarthestFirst is the fastest one and thus has the smallest latency. Among all the classifier services, Weka REPTree Classifier has the smallest latency. Weka Apriori and Tertius Associator failed because Lung Cancer dataset is not nominal data type. Throughput value varies among different services. Since we consider failure invocations for the Reliability, we see different Reliability values such as $100 \%, 42.85 \%$, etc.

Table 4.16 shows the average QoS values of all SaaS on one IaaS (Azure-1). The QoS values are averaged on all datasets.

From this table, we can see that the Reliability values vary when we average QoS values on datasets. Weka DBSCAN clustering services failed for all the datasets. Weka Apriori Associator and Weka Tertius Associator have less reliability than any other software services. A few services have $100 \%$ reliability, which means they work properly for all the datasets. We can also see the variation of latency, response time and throughput. We ran our simulation in different timeframe, for example, pick hour and off-pick hour and we also chose different cloud server locations. Sometimes we set wrong parameters for clustering services. Sometimes we observed the network problem to communicate with the cloud server from the client application. These influential factors make the difference of the QoS values and these differences affect throughput directly because throughput value depends on service output, response time and latency. 
Table 4.16: Average QoS values of all SaaS on one IaaS (Azure-1)

\begin{tabular}{|c|c|c|c|c|}
\hline Service Name & Latency (sec) & Response Time (sec) & Reliability & Throughput \\
\hline Weka EM Clustering & 2.235 & 7.535 & $83.33 \%$ & 3.235 \\
\hline Weka Hierarchical Clustering & 0.173 & 3.706 & $16.67 \%$ & 1.643 \\
\hline Weka Filtered Clustering & 0.251 & 3.9829 & $83.33 \%$ & 5.458 \\
\hline Weka CLOPE Clustering & 0.295 & 4.8698 & $16.67 \%$ & 0.839 \\
\hline Weka FarthestFirst Clustering & 0.445 & 3.697 & $100 \%$ & 1.493 \\
\hline Weka SimpleKMeans Clustering & 3.165 & 8.067 & $100 \%$ & 3.680 \\
\hline Weka Cobweb Clustering & 0.093 & 4.112 & $33.33 \%$ & 2.361 \\
\hline Weka sIB Clustering & 0.537 & 4.449 & $16.67 \%$ & 1.864 \\
\hline Weka XMeans Clustering & 0.182 & 4.292 & $66.67 \%$ & 4.366 \\
\hline \multicolumn{5}{|l|}{ Weka DBSCAN Clustering } \\
\hline Apache KMeansPlusPlus Clustering & 0.076 & 4.428 & $50 \%$ & 4.179 \\
\hline Apache DBSCAN Clustering & 0.082 & 4.274 & $50 \%$ & 0.073 \\
\hline R Hierarchical Clustering & 0.333 & 4.046 & $50 \%$ & 12.595 \\
\hline R KMeans Clustering & 0.172 & 3.436 & $50 \%$ & 11.955 \\
\hline Weka BFTree Classifier & 0.762 & 4.590 & $83.33 \%$ & 1.768 \\
\hline Weka AdaBoostM1 Classifier & 0.436 & 3.103 & $100 \%$ & 1.893 \\
\hline Weka REPTree Classifier & 0.215 & 3.507 & $100 \%$ & 1.819 \\
\hline Weka LMT Classifier & 0.302 & 3.052 & $83.33 \%$ & 2.073 \\
\hline Weka RandomTree Classifier & 0.294 & 4.014 & $100 \%$ & 2.001 \\
\hline Weka RandomForest Classifier & 0.324 & 4.101 & $100 \%$ & 1.903 \\
\hline Weka Apriori Associator & 0.057 & 3.831 & $16.67 \%$ & 1.873 \\
\hline Weka Tertius Associator & 7.336 & 11.061 & $16.67 \%$ & 0.548 \\
\hline
\end{tabular}




\subsection{Summary}

In this chapter, we explained our implementation details, our programming environment, our user interfaces, data mining packages, and infrastructure configurations. We selected three algorithm providers, two cloud service providers and six UCI datasets. We chose our datasets based on different data properties such as dimension, size, missing values, data types (numeric data, string data and combined data etc.).

From our collected QoS values, we could see some services failed to process the file. The reason of failure could lie on dataset, algorithm implemented and parameters that users send to the service. We see some services such as Weka DBSCAN, Weka CLOPE, and Weka OPTICES clustering services are directly affected by the parameter values. R clustering and Apache clustering services are affected by the data type because these two provider's algorithms support only numeric datasets. Some services only support nominal data type, such as Weka CLOPE clustering service. Sometimes we see QoS values vary a lot due to the network communication from client system to the cloud server. 


\section{CHAPTER 5}

\section{CONCLUSION AND FUTURE WORK}

\subsection{Conclusion}

QoS-based service selection in the cloud environment could be a big challenge now-adays, especially for data analytic services. In the cloud environment, different users follow different strategies for data analytic service selection. QoS properties such as availability, latency, response time, reliability and throughput are some influential factors for selecting these services. The values of these properties for data analytic services could vary for different datasets, and they also depend on how they are implemented and where and how they are hosted. Although various approaches have been studied and proposed, there is no proper benchmarking tool that will record the end-to-end QoS values for these software services when they are running on different datasets and hosted in different infrastructure servers.

Our contributions are listed below:

- We designed and implemented a cloud-based framework to build a testbed that could be used for collecting end-to-end QoS values from data analytic services. These QoS values are helpful for comparing different selection algorithm proposed by different researchers.

- We implemented a proof of concept prototype system where we chose data mining algorithms from different providers to develop data analytic services as representative services. We found that provider, functionality and implemented algorithm are some SaaS related factors that impact QoS values. We hosted our 
developed data analytic services in different cloud infrastructure services with different configurations in different locations. We found that provider, location and configuration are some IaaS related factors that impact QoS values.

\subsection{Future Works}

QoS based service selection is expanding and growing day by day in different sectors including research community, government organization and private industries. There are a few directions we would like to work on in the future.

- Firstly, we could expand our current system to a fully functioning cloud marketplace with more functionalities.

- Secondly, in this work, we only consider five QoS properties, and in the future we could include more QoS properties.

- Thirdly, right now we only support RESTful services, and in the future we have plan to include the support to SOAP based services as well because they are still used in many organizations.

- We plan to introduce other services that are not based on REST.

- Lastly, we could try more SaaS services, IaaS instances and more datasets to collect a bigger sized QoS dataset to share with the research community. 


\section{REFERENCES}

[1] Service (systems architecture). Available at: http://en.wikipedia.org/wiki/service(systems architecture), last accessed on August 2015.

[2] OASIS SOA Reference Model. Available at: https://www.oasis-open.org/committees/tc home.php?wg_abbrev=soa-rm, last accessed on August 2015.

[3] R. Buyya, C. S. Yeo, S. Venugopal, J. Broberg, and I. Brandic, "Cloud computing and emerging IT platforms: vision, hype, and reality for delivering computing as the 5th utility", 2009 Future Generation Computer Systems, pp. 599-616.

[4] J. Yan and J. Piao, "Towards QoS-based web services discovery," 2009 Service-Oriented Computing ICSOC 2008 Workshops, pp. 200-210.

[5] E. Al-Masri and Q. H. Mahmoud, "QoS-based Discovery and Ranking of Web Services", 2007 16th International Conference on Computer Communications and Networks (ICCCN), pp. 529-534.

[6] Y. Liu, A. H. Ngu and L. Z. Zeng, "QoS Computation and Policing in Dynamic Web Service Selection", 2004 13th International World Wide Web Conference, pp. 66-73.

[7] Q. He, J. Han, Y. Yang, J. Grundy and H. Jin, "QoS-driven service selection for multitenant SaaS", 2012 IEEE Fifth International Conference on Cloud Computing, pp. 566-573. 

services under multi-criteria matching," 2009 12th International Conference on Extending Database Technology: Advances in Database Technology, pp. 898-909.

[9] Q.MA, H. WANG, Y. LI, G. XIE and F. LIU , "A Semantic QoS-aware Discovery Framework for Web Services", 2008 IEEE International Conference on Web Services, pp. 129136.

[10] R. Madaleno and R. Neto Marinheiro, "Identification and ranking of relevant criteria for the selection of Software as a Service", 2013 8th Iberian Conference on Information Systems and Technologies (CISTI), pp. 1-6.

[11] Y. M. Afify, I. F. Moawad, N. L. Badr and M.F. Tolba, "A Semantic-based Software-asa-Service (SaaS) Discovery and Selection System", 2013 8th International Conference on Computer Engineering \& Systems (ICCES), pp. 57-63.

[12] S. Le, H. Dong, H. F.K, H. O.K, J. Ma and Y. Zhang, "Multicriteria Decision Making with Fuzziness and Criteria Interdependence in Cloud Service Selection", 2014 IEEE International Conference on Fuzzy Systems (FUZZ-IEEE), pp. 1929-1936.

[13] R. K. Lomotey and R. Deters, "Analytics-as-a-Service (AaaS) Tool for Unstructured Data Mining", 2014 IEEE International Conference on Cloud Engineering, pp. 319- 324.

[14] F. Zulkernine, M. Bauer and A.Aboulnaga, "Towards Cloud-based Analytics-as-aService (CLAaaS) for Big Data Analytics in the Cloud", 2013 IEEE International Congress on Big Data, pp. 62-69.

[15] IBM Research “Analytics-as-a-Service Platform”. Available at: http://researcher.ibm.com 
/researcher/view_project.php?id=3992, last accessed on August 2015.

[16] The QWS Dataset. Available at: http://www.uoguelph.ca/ qmahmoud/qws/, last accessed on August 2015.

[17] WS-Dream Dataset. Available at: http://www.wsdream.net/dataset.html, last accessed on August 2015.

[18] The WEKA toolkit, University of Waikato. Available at: http://www.cs.waikato.ac.nz/ml/ weka/, last accessed on August 2015.

[19] The R Project for Statistical Computing. Available at: http://www.r-project.org/, last accessed on August 2015.

[20] Apache Commons Math Machine Learning. Available at: http://commons.apache.org/ proper/commons-math/userguide/ml.html, last accessed on August 2015.

[21] K. Kritikos and D. Plexousakis, "Mixed-Integer Programming for QoS-based Web Service Matchmaking”, 2009 IEEE Transactions on Services Computing, pp. 122-139.

[22] C.W. Hang and M.P. Singh, "From Quality to Utility: Adaptive Service Selection Framework”, 2010 International Conference on Service Oriented Computing, pp. 456-470.

[23] R.J.R. Raj and T. Sasipraba, "Web service selection based on QoS Constraints", 2010 Trends in Information Sciences \& Computing (TISC), pp. 156-162.

[24] V. X. Tran, H. Tsuji and R. Masuda, "A new QoS ontology and its QoS-based ranking algorithm for Web services," 2009 Simulation Modelling Practice and Theory, pp. 1378-1398. 
[25] L. Qu, Y. Wang and O. M.A, "Cloud Service Selection Based on the Aggregation of User Feedback and Quantitative Performance Assessment", 2013 IEEE International Conference on Services Computing (SCC), pp. 152-159.

[26] L. Sun, H. Dong, F.K. Hussain, O.K. Hussain, J. Ma and Y. Zhang, "A Hybrid Fuzzy Framework for Cloud Service Selection", 2014 IEEE International Conference on Web Services, pp. 313-320.

[27] M. Tajvidi, R. Ranjan, J. Kolodziej and L. Wang, "Fuzzy cloud service selection framework", 2014 IEEE 3rd International Conference on Cloud Networking (CloudNet), pp. 443-448.

[28] A.Kr Mandal, S. Changder and A. Sarkar, " Selection of Services for Data-Centric Cloud Applications: A QoS Based Approach", 2013 Second International Conference on Advanced Computing, Networking and Security, pp. 102-107.

[29] M. Sun, T. Zang, X. Xu and R. Wang, "Consumer-Centered Cloud Services Selection Using AHP", 2013 International Conference on Service Science, pp. 1 - 6.

[30] R. Karim, C. Ding and A. Miri , "An End-To-End QoS Mapping Approach for Cloud Service Selection", 2013 IEEE Ninth World Congress on Services, pp. 341-348.

[31] H. Li and J. Jeng, "CCMarketplace: A Marketplace Model for a Hybrid Cloud", 2010 Conference of the Center for Advanced Studies on Collaborative Research, pp. 174-183.

[32] T. Heninger, A. Singh, V. Singh, T. Wies and D. Zufferey, "A Marketplace for Cloud Resources", 2010 International Conference of Embedded Systems, pp. 1-7. 
[33] S. Chang, "Application Marketplace as a Service - A Reference Architecture for Application Marketplace Service", 2010 International Conference on Cloud and Internet Computing (3PGCIC), pp.186-192.

[34] A. Menychtas, A. Gatzioura and T. Varvarigou, "A Business Resolution Engine for Cloud Marketplaces”, 2011 Third IEEE International Conference on Coud Computing Technology and Science, pp. 462-46.

[35] O. Krieger, P. McGachey and A. Kanevsky, "Enabling a Marketplace of Clouds: VMware's vCloud Director", 2010 Operating Systems Review, pp. 103-114.

[36] S. Song, M. M. Hassan and E. Huh, "A Novel Cloud Market Infrastructure for Trading Service", 2009 International Conference on Computational Science and Its Applications, pp. 4450.

[37] Cloud Broker. Available at: http://searchcloudprovider.techtarget.com/definition/cloudbroker, last accessed on August 2015.

[38] S. Sundareswaran, A. Squicciarini, and D. Lin, "A Brokerage-Based Approach for Cloud Service Selection", Cloud Computing (CLOUD), 2012 IEEE 5th International Conference, pp.558-565.

[39] P.Pawluk, B. Simmons, M. Smit, M. Litoiu and S.Mankovski, "Introducing STRATOS: A Cloud Broker Service", 2012 IEEE Fifth International Conference on Cloud Computing, pp. 891-898.

[40] V. Cardellini and S.Iannucci, "Designing a Broker for QoS-driven Runtime Adaptation of SOA Applications", 2010 IEEE International Conference on Web Services, pp. 504-511. 
[41] F. Ye, Z.Wang, F. Zhou, Y.Wang and Y. Zhou, "Cloud-Based Big Data Mining \& Analyzing Services Platform Integrating R", 2013 International Conference on Advanced Cloud and Big Data (CBD), pp.147-151.

[42] T.Chen, J. Chen and B. Zhou, "A System for Parallel Data Mining Service on Cloud", 2012 Second International Conference on Cloud and Green Computing (CGC), pp. 329-330.

[43] Web service. Available at: http://www.w3.org/TR/2004/NOTE-ws-gloss-20040211/ \#webservice, last accessed on August 2015.

[44] Roy T. Fielding and Richard N. Taylor, "Principled Design of the Modern Web Architecture", 2000 22nd international conference on Software engineering, pp.407-416.

[45] Microsoft Azure. Available at: http://blogs.microsoft.com/blog/2010/02/01/windowsazure-general-availability/, last accessed on August 2015.

[46] UCI Machine Learning Repository. Available at: https://archive.ics.uci.edu/ml/ datasets.html, last accessed on August 2015.

[47] Richard K. Lomotey and R. Deters, "Analytics-as-a-Service (AaaS) Tool for Unstructured Data Mining", 2014 IEEE International conference on Cloud Engineering, pp. 319324.

[48] K. Park, M. C. Nguyen and H.Won, "Web-based Collaborative Big Data Analytics on Big Data as a Service platform", 2014 IEEE International conference on Big Data, pp. 564-567. 